\title{
Star formation losses due to tidal debris in "hierarchical" galaxy formation
}

\author{
B. F. Roukema ${ }^{1,2,3,4, \star}$, S. Ninin ${ }^{1}$, J. Devriendt ${ }^{1}$, F. R. Bouchet ${ }^{1}$, B. Guiderdoni ${ }^{1}$, and G. A. Mamon ${ }^{1,5}$ \\ 1 Institut d'Astrophysique de Paris, 98bis Bld. Arago, 75014 Paris, France \\ e-mail: ninin@iap.fr; devriend@iap.fr; bouchet@iap.fr; guider@iap.fr; gam@iap.fr \\ 2 Observatoire astronomique de Strasbourg, CNRS, 11 rue de l'Université, 67000 Strasbourg, France \\ 3 Nicolaus Copernicus Astronomical Center, ul. Bartycka 18, 00-716 Warsaw, Poland \\ 4 Inter-University Centre for Astronomy and Astrophysics, Post Bag 4, Ganeshkhind, Pune, 411 007, India \\ 5 DAEC, Observatoire de Paris-Meudon, 5 place Jules Janssen, 92195 Meudon Cedex, France
}

Received 27 January 2000 / Accepted 7 May 2001

\begin{abstract}
N$-body studies have previously shown that the bottom-up hierarchical formation of dark matter haloes is not as monotonic as implicitly assumed in the Press-Schechter formalism. During and following halo mergers, matter can be ejected into tidal tails, shells or low density "atmospheres" outside of the successor haloes' virialisation radii (or group-finder outermost radii). The implications that the possible truncation of star formation in this tidal "debris" may have for observational galaxy statistics are examined here using the ARFus N-body plus semi-analytical galaxy modelling software for standard star formation hypotheses. In the $N$-body simulations studied, the debris typically remains close to the successor halo and falls back into the successor haloes given sufficient time. A maximum debris loss of around $16 \%$ is found for redshift intervals of around $\Delta z=0.4$ at $z \sim 1$, with little dependence on the matter density parameter $\Omega_{0}$ and the cosmological constant $\lambda_{0}$. Upper and lower bounds on stellar losses implied by a given set of $N$-body simulation output data can be investigated by choice of the merging/identity criterion of haloes between successive $N$-body simulation output times. A median merging/identity criterion is defined and used to deduce an upper estimate of possible star formation and stellar population losses. A largest successor merging/identity criterion is defined to deduce an estimate which minimises stellar losses. The losses for star formation and luminosity functions are strongest for low luminosity galaxies - a likely consequence of the fact that the debris fraction is highest for low mass haloes - and at intermediate redshifts $(1 \lesssim z \lesssim 3)$. The losses in both cases are mostly around $10 \%-30 \%$, have some dependence on $\Omega_{0}$ and negligible dependence on $\lambda_{0}$. This upper bound on likely losses in star formation rates and stellar populations is smaller than the uncertainties in estimates of corresponding observational parameters. Hence, it may not be urgent to include a correction for this in Press-Schechter based galaxy formation models, except when statistics regarding dwarf galaxies are under study.
\end{abstract}

Key words. galaxies: formation - galaxies: luminosity function - galaxies: interactions - galaxies: irregular cosmology: theory - methods: numerical

\section{Introduction}

An updated version of the galaxy formation modelling technique of Roukema et al. (1997) is used here to examine one of the consequences of the fact that "(bottom-up) hierarchical" galaxy formation is not strictly bottom-up hierarchical.

A bottom-up hierarchical galaxy formation scenario is (bottom-up) hierarchical in the sense that small fluctuations in an otherwise constant matter density fluid initially

Send offprint requests to: B. F. Roukema,

e-mail: boud.roukema@obspm.fr

* Present address: DARC/LUTH, Observatoire de ParisMeudon, 5 place Jules Janssen, 92195 Meudon Cedex, France. collapse on small length and mass scales, forming low mass dark matter haloes, which then merge together to form successively higher and higher mass haloes ${ }^{1}$. However, when haloes merge, significant fractions of the matter in pre-merger haloes are thrown out in tidal tails, bridges and other forms of debris. This has been well known since the review and further development of gravity-only $N$-body simulations of close interactions of gravitating disks by Toomre \& Toomre (1972). Indeed, observations suggest that at least some of this debris may form

\footnotetext{
1 The word "halo" in this context is independent of the word "halo" sometimes used to describe a spherically symmetric stellar component of the Galaxy, or of other galaxies.
} 
visible galaxies which are dynamically bound entities (Duc \& Mirabel 1994; Duc et al. 1997). Thus, the bottom-up hierarchy is broken.

For recent discussions of tidal destruction of haloes in $N$-body simulations see, e.g. Klypin et al. (1999) or Figs. 7 and 8 of Kravtsov \& Klypin (1999). For a debate on the conditions required for the formation of tidal tail galaxies, see Dubinski et al. (1996), Mihos et al. (1998) and Springel \& White (1999).

Ejection of matter into tidal debris is a top-down process. So, bottom-up hierarchical halo formation is not strictly hierarchical: some material is (at least temporarily) thrown out of (or "down") the bottom-up hierarchy.

Bottom-up hierarchical ${ }^{2}$ galaxy formation models have been modelled by several different techniques (Roukema et al. 1993; Kauffmann et al. 1993).

The primary output of hierarchical galaxy formation models that can be compared with observations is the modelling of the stellar components of the haloes, i.e. galaxies. Stellar population models in hierarchical models depend on star formation, or more precisely on star formation rate history trees. In hierarchical galaxy formation models, star formation rate histories are physically best represented as trees, since any single galaxy is (in general) the result of a history tree of galaxy merging (Roukema et al. 1993; Lacey \& Cole 1993; Kauffmann et al. 1993).

By assuming that haloes collapse in an idealised, monotonic, spherically symmetric way, and that merging of haloes proceeds monotonically in the sense that once matter is considered collapsed (virialised) in a halo, it remains collapsed when the halo merges with other haloes, Kauffmann et al. (1993) used the probabilistic formulae of the Press-Schechter formalism (e.g. Press \& Schechter 1974; Lacey \& Cole 1993) to generate merging history trees of haloes, and followed through to merging trees of galaxies and to synthetic values of galaxy observables such as apparent magnitudes.

Roukema et al. (1993) generated merging history trees without making this physical assumption by directly detecting haloes in pure gravity $N$-body simulations, and then followed through to galaxy merging trees and apparent magnitudes.

What differences can arise by avoiding the monotonic collapse assumption of the Press-Schechter formalism? The star formation rates in the material ejected as tidal debris are unlikely to be the same as those which would be expected if the material fell in smoothly according to the monotonicity assumption.

Could the monotonic collapse assumption be a good approximation if

(i) the ejected material remains gravitationally bound to the merging system, i.e., it forms a low-density "atmosphere" around the halo resulting from the merger (the merger product), and/or

(ii) it falls back within a dynamical time?

\footnotetext{
2 Hereafter, "hierarchical" means "bottom-up hierarchical" unless otherwise specified.
}

In both these cases, the physical conditions of the cold gas fraction of the debris are likely to be temporarily different from those of the gas which falls directly into a disk at the centre of the main merger product without passing through this "debris" phase. Hence, the star formation rate, which is observed to depend on density (e.g. Kennicutt 1998), is likely to be different for matter which passes through a "debris" stage compared to matter falling smoothly into a disk.

The difference in the star formation rate of debris relative to that for the monotonic infall case could, in principle, be either negative or positive. Gas in low-density streams or atmospheres is likely to form stars more slowly than in the disk, whereas gas inside newly condensed tidal dwarf haloes may be of higher density than in the disk and form stars much more rapidly.

However, stars formed in the debris are likely either to dynamically become part of the main merger product, or to observationally be counted in the integrated luminosities and colours of the main products in faint galaxy catalogues. It may then be a valid approximation (for some galaxy statistics) to ignore the violation of hierarchy, i.e. to assume that the debris provides dark matter (nonbaryonic plus baryonic) which is available for gas cooling and star formation, as if they were part of the virialised successor halo, and as if they were not thrown out of the successor halo.

As mentioned above, this is the assumption implicitly made in semi-analytical galaxy formation models based either on Press-Schechter probability estimates of hierarchical halo formation (e.g. Kauffmann et al. 1993; Guiderdoni et al. 1998) or on extrapolation from linear density fields (the block model of Cole et al. 1994; the multi-cell-merging model of Rodrigues \& Thomas 1996, see also Nagashima \& Gouda 1998; Lanzoni et al. 2000). Following the implicit assumption of monotonic, spherical infall, assumptions on gas cooling, star formation rates and gas reheating are made, in order to derive observational properties of galaxies.

The two limiting cases of the fate of debris which should bound other possibilities can be stated in simple terms as:

(a) most debris either becomes dynamically independent or is "effectively independent" in that it takes a large fraction of a Hubble time to fall back into the virialised part of the halo product and does not contribute stars to the central galaxy from an observer's point of view; or

(b) most debris either forms an "atmosphere" close to the central galaxy or falls back into the central galaxy relatively quickly so that the mononotonic, spherical infall assumption is a good approximation and star formation happens as if infall really were monotonic.

How much effect could the difference between (a) and (b) have on galaxy statistics such as the luminosity function? What would be the decrease in star formation rates and 
how much could this affect the present-day luminosity function?

These questions can be investigated by looking at the effect that the merger/identity criterion used in merging history tree galaxy simulations can have on the luminosity function.

This is the purpose of this paper. An $N$-body plus semi-analytical galaxy formation modelling technique (Roukema et al. 1993, 1997) is used to study the effects that tidal debris could have on global galaxy statistics. The technique used is that of combining gravityonly $N$-body simulations with semi-analytical formulae (Roukema et al. 1993, 1997), updated from the Roukema et al. (1997) version by the creation of a multiple-galaxyper-halo merging history tree using the dynamical friction formalism of Lacey \& Cole (1993), and the inclusion of assumptions on gas cooling, star formation rates and gas reheating as in Kauffmann et al. (1993). A brief description of the updated model, which is publicly available as the ARFus software package, is provided in the Appendix.

Use of this galaxy formation model enables investigation of the negative effects that the passage of matter through a "debris" stage could have on star formation rates and luminosities.

In Roukema et al. (1997), the problems of tidal debris were noted and a simple, robust "merger/identity" criterion for deciding how to identify halo progenitors and successors between successive output time steps was adopted. In Sect. 2, ways to modify this merger/identity criterion and the way in which these modifications enable investigation of the limiting cases (a) and (b) for the effects of debris on galaxy formation are presented. In Sect. 3, effects on galaxy statistics are shown. The results are discussed and conclusions provided in Sect. 4.

Only one cosmological model (the perturbed Friedmann-Lemaître model) is considered here. The Hubble constant is parametrised here as $h \equiv H_{0} / 100 \mathrm{~km} \mathrm{~s}^{-1} \mathrm{Mpc}^{-1}$. Three options for the other metric parameters (the present values of the density parameter, $\Omega_{0}$, and of the dimensionless cosmological constant, $\lambda_{0}$ ) are considered (see Table 2).

\section{The merger/identity criterion and debris}

The principle of combining $N$-body simulations with semianalytical techniques, in which a series of output times from the simulations are used, was presented in Roukema et al. $(1993,1997)$ and has more recently been adopted by Kauffmann et al. (1999).

This should not be confused with the technique of applying the extended Press-Schechter formalism to create merging histories for haloes detected in one $N$-body simulation output time (Kauffmann et al. 1997; Governato et al. 1998; Benson et al. 1999). The latter could possibly be described as using $N$-body simulations to "normalise" the Press-Schechter technique of generating a set of merging history trees, as opposed to using the $N$-body simulations to generate the trees directly.
The precise algorithm for constructing the halo merging history tree is described in Sect. 2.1.3 of Roukema et al. (1997). In that paper, haloes between two successive $N$-body simulation output times $\left(t_{i}, t_{i+1}\right)$ were considered to have a merger/identity link if at least $P=50 \%$ of the particles in a halo at $t_{i}$ were present in a halo at $t_{i+1}$. This is the $P=50 \%$ criterion. With this criterion, and for the same halo detection method (group finder algorithm) at each output time, some haloes were found not to have successors. That is, there are some haloes for which more than $50 \%$ of their material forms tails, cusps, shells and low density envelopes outside of the group-finder defined boundaries of haloes.

Given this criterion, typically $P \sim 75-90 \%$ of a halo at $t_{i}$ is present in a halo at $t_{i+1}$ (Table 3 , Roukema et al.). This fraction depends on the group finder density detection threshold and on the slope of the power spectrum of density perturbations used in the $N$-body simulation.

Kauffmann et al. (1999) adopted a slightly modified version of this $P=50 \%$ criterion as their merger/identity criterion, but haloes failing the criterion were considered to generate "lost" galaxies which could later on either merge with a central galaxy or become a satellite galaxy if the criterion were satisfied at a later $N$-body output time.

Both the original criterion of Roukema et al. and that of Kauffmann et al. are bracketted by the following two limiting cases.

\subsection{The "median" merger/identity criterion for modelling case (a): The loss of debris}

By terminating and (in effect) removing haloes that fail the $P=50 \%$ criterion from later times in the halo merging history tree, the ejection of debris is implicitly modelled. However, the effect is in fact exaggerated, since stars previously formed in haloes that fail the $P=50 \%$ criterion no longer contribute to the stellar population of any galaxy, neither in the present timestep (as they are no longer in haloes) nor later (as they are not added to future haloes). This is useful for obtaining an upper bound to the loss in star formation and in luminosity that would be caused by the debris stage, i.e. to bound (a) from above.

However, the choice of $P=50 \%$ as the minimum merger/identity percentage is an arbitrary (though simple) choice designed to guarantee at most a single successor halo, which does not have any other physical motivation. A less arbitrary choice for a merger/identity criterion should be one that both

(i) excludes haloes of which the fraction thrown into debris is higher than "typical", and

(ii) retains the property of guaranteeing a single successor halo.

The choice of the successor halo that contains the highest fraction of a progenitor halo is sufficient to satisfy (ii), except for the physically unlikely but numerically possible case where a progenitor halo contributes two equal (rational) fractions (e.g. 11/30ths) to two successor haloes. 
Table 1. Summary of the different merging/identity criterion choices, where $P_{\min }$ is the minimum fraction of particles of a halo at time $t_{i}$ present in a halo at time $t_{i+1}$ in order for a merging/identity link to be defined between the two haloes, and $N$ is the number of particles in the halo at $t_{i+1}$. The $50 \%$ criterion of Kauffmann et al. (1999) differs slightly from the $50 \%$ criterion of Roukema et al. (1997), and allows recuperation of "lost" galaxies at later time steps.

\begin{tabular}{ccc}
\hline$P_{\min }$ & name & comments \\
0.5 & $50 \%$ & Roukema et al. (1997) \\
0.5 & - & Kauffmann et al. (1999) \\
$2 P_{\text {med }}-1$ & median & loss of debris; this paper \\
$1 / N$ & largest successor & monotonic infall; this paper \\
\hline
\end{tabular}

For property $(\mathrm{i})$, the median merging fraction $\left(P_{\text {med }}\right)$ can be used to define a "typical" merging fraction, so that mergers for which the debris fraction is "much higher" than the median debris fraction, i.e. $1-P \gg 1-P_{\text {med }}$, can be excluded.

An obvious way to define "much higher" is to note that all mergers with percentages higher than the median $\left(P>P_{\text {med }}\right)$ ought to be included by the criterion. By choosing an exclusion percentage which is as far below the median as $100 \%$ is higher than the median, i.e. for which

$1-P_{\mathrm{med}}=P_{\mathrm{med}}-P \quad \Leftrightarrow \quad P=2 P_{\mathrm{med}}-1$,

a symmetry about the (original) median is obtained.

This defines the "median" merger/identity criterion: a halo at $t_{i}$ is linked with the halo at $t_{i+1}$ that contains the largest fraction of particles from the halo at $t_{i}$, as long as that successor halo (at $t_{i+1}$ ) contains more than a fraction $P=\left(2 P_{\text {med }}-1\right)$ of the particles in the halo at $t_{i}$, irrespective of whether the merging fraction is greater than $50 \%$ or not.

The median fraction $P_{\text {med }}$ is calculated from the full set of output time pairs for a given $N$-body simulation, and for reasons of dynamical consistency is likely to remain well above $50 \%$. The value $P=\left(2 P_{\text {med }}-1\right)$ may, in principle, fall below $50 \%$. If it is above $50 \%$, then the rare cases of equal contributions to multiple successor haloes mentioned above are automatically excluded.

The algorithm in Sect. 2.1.3 of Roukema et al. (1997) can easily be modified in order to implement this (and the following) merger/identity criterion, by sorting the two arrays of halo numbers $a_{i}, a_{i+1}$ in such a way that the array $a_{i}$ (instead of the array $a_{i+1}$ ) becomes a non-decreasing arithmetical sequence.

\subsection{The "largest successor" criterion for modelling case (b): Smooth, monotonic infall}

For the other limiting case, in which debris is to be included as much as possible in successor galaxies, the "largest successor" merger/identity criterion is defined as follows.

Each halo at $t_{i}$ has a link made with the halo at $t_{i+1}$ that contains the largest fraction of the particles in the halo at $t_{i}$, even if this is fraction is less than $50 \%$. This requires that at least $N=1$ particle in a halo at $t_{i}$ is present in a halo at $t_{i+1}$ in order for the halo at $t_{i}$ to have a successor. This is usually the case.

As in the case of the $P=50 \%$ and median criteria, a halo has at most one successor with the largest successor criterion, since, by definition, only one halo at $t_{i+1}$ can have the largest fraction of particles of the halo at $t_{i}$, apart from the unlikely case of equal rational fractions, as mentioned above. In the $N$-body simulations studied here, the latter case (double largest successor, with a merging percentage of less than $\sim 20 \%$ ), occurs in about $0.1 \%$ of cases, and an arbitrary choice between the two successors is made.

The different merging/identity criteria are summarised in Table 1.

\subsection{N-body simulations and group-finding}

The $N$-body simulations used are particle-mesh $N=$ $2 \times 10^{6}$ simulations in a comoving "periodic box" of size $32 h^{-1}$ Mpc (i.e. the cosmology is hypertoroidal, e.g. Farrar \& Melott 1990; Luminet \& Roukema 1999). Three choices of metric parameters are used: $\left(\Omega_{0}=1, \lambda_{0}=\right.$ $0, h=0.65),\left(\Omega_{0}=0.3, \lambda_{0}=0.7, h=0.65\right)$ and $\left(\Omega_{0}=\right.$ $\left.0.3, \lambda_{0}=0.0, h=0.65\right)$. The baryon density is $\Omega_{\mathrm{b}}=0.05$. The initial perturbation spectrum is a CDM-like spectrum (Eq. (2) of Davis et al. 1985, where $\Gamma \equiv \Omega_{0} h$ is substituted for $\Omega_{0} h$ ), where $\Gamma=0.3$ and the normalisation at $8 h^{-1} \mathrm{Mpc}$ is $\sigma_{8}=0.6$.

Haloes are detected using the University of Washington friends-of-friends (FOF) group-finder ${ }^{3}$ with a friendship distance of $b=0.2$ times the mean interparticle spacing and a minimum of 30 particles per halo.

\subsection{Star formation assumptions for comparing the median and largest successor merging/identity criteria}

To see the way that the median (Sect. 2.1) and largest successor (Sect. 2.2) merging/identity criteria may respectively test the limiting cases (a) and (b) (Sect. 1), the halo merging history trees resulting from the different criteria can be combined with a semi-analytical formalism to derive star formation and luminosity statistics. This is done here using the galaxy formation model ARFus, updated from Roukema et al. (1997), which applies semi-analytical star formation hypotheses to a multiple-galaxy-per-halo merging history tree. ARFUS-V0.03 is used in the present study, with the simple stellar populations (for solar

\footnotetext{
${ }^{3}$ Available at http://www-hpcc. astro. washington. edu/tools/
} 
metallicity and a Salpeter initial mass function) of Devriendt et al. (1999).

The model presented in Roukema et al. (1997) represented maximal galaxy merging, i.e. there was a 1:1 correspondence between haloes and galaxies and the galaxy merging history tree was identical to the halo merging history tree. In the updated model, a galaxy merging tree is derived from the halo merging tree by following Lacey \& Cole's (1993) suggestion:

(i) when two haloes merge, the galaxy in the more massive halo becomes the central galaxy of the successor halo, while the galaxy in the less massive halo becomes a "satellite" galaxy, and

(ii) the satellite galaxy is allowed to spiral in to the centre of the parent halo by dynamical friction.

The dynamical friction merger time is estimated via Eq. (4.2) of Lacey \& Cole (1993), including the effect of orbital eccentricity.

Note that the merger time of the satellite galaxy may be greater than the interval between $N$-body simulation output times. This leads to an increased complexity in both calculation and storage of the combined galaxy plus halo merging history tree. See the Appendix (Sect. 5.2) for the algorithm adopted in ARFus-V0.03.

As in Kauffmann et al. (1993), cooling functions and singular isothermal total matter density profiles are used to calculate cooling radii within which gas cools, a star formation rate depending on the amount of cold gas and the dynamical time is applied, and some gas is reheated by energy from supernovae. The different options (a) and (b) for the halo merger/identity criterion lead automatically to different amounts of gas available for star formation, and to loss of stars from debris.

The cooling and heating rate formulae of Kauffmann et al. (1993) are adopted here, with a standard value of the reheating efficiency free parameter, $\varepsilon=10 \%$ (Eq. (9) of Kauffmann et al. 1993) and the cooling functions of Sutherland \& Dopita (1993). The star formation rate is

$\frac{\mathrm{d} M_{*}}{\mathrm{~d} t}=\alpha \frac{M_{\mathrm{cold}}}{t_{\mathrm{dyn}}}$,

where $M_{*}$ is the stellar mass, $\alpha$ is a star formation rate efficiency free parameter normally set to $\alpha=1, M_{\text {cold }}$ is the mass of cold gas and $t_{\text {dyn }}$ is the dynamical time of the parent halo at the earlier $N$-body simulation output time of the output time pair.

See the Appendix for more comments on ARFus, or the electronic site of ARFus (address in Appendix) for full documentation.

\section{Results}

\subsection{Merging fractions}

The relevance of the merging/identity criteria can be verified by studying the following questions:

(i) How does the fraction of debris depend on halo mass?

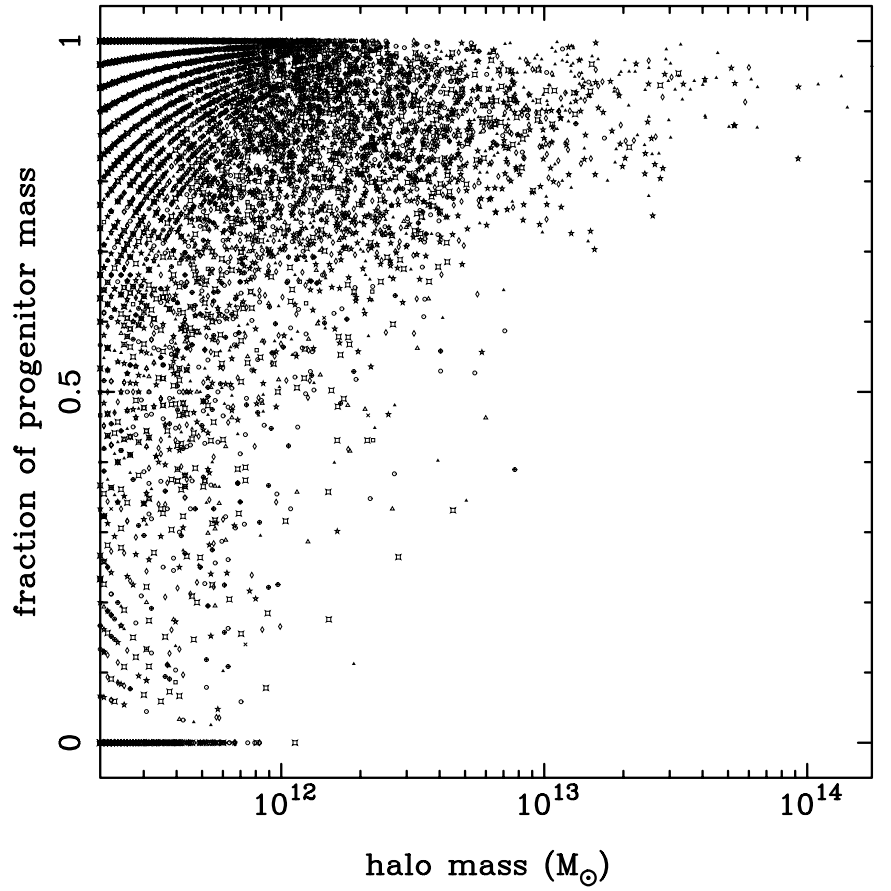

Fig. 1. The fraction $P$ of a progenitor halo present in a successor halo between two $N$-body simulation output times separated by $\Delta z=0.5$, for successive output time pairs from $z=6$ to $z=0$, for the $\left(\Omega_{0}=1, \lambda_{0}=0\right)$ CDM-like simulation detailed in Sect. 2.3, as a function of progenitor halo mass. Different symbols indicate different output time pairs, but to avoid crowding are too small to be easily distinguishable (the "squares with pointy corners" representing the interval $z \in[2.5,2.0]$ may be distinguishable, and possibly also the solid triangles for $z \in[0.5,0.0])$. The value of $P$ is unity for the assumption of monotonic, spherical infall. Note that the effects of discreteness show up as patterns at the left-hand edge of the figure, even though a minimum of 30 particles is required per halo. The points with $P=0$ represent haloes for which no successor at all exists in the output time following by $\Delta z=0.5$.

(ii) How do they depend on the output time (redshift) and the output time (redshift) intervals?

(iii) How far from the central halo is the debris located?

(iv) Does the debris just form loose atmospheres and tidal tails, or does it revirialise and form tidal dwarf haloes/galaxies?

Figures 1-3 and Table 2 provide responses to these questions for some typical $N$-body simulations.

\subsection{1. (i) How does the fraction of debris depend on halo mass?}

Figure 1 clearly shows that for a flat, critical density CDM-like universe, the low mass haloes are more disrupted than the high mass haloes. This is physically reasonable, since it is more likely that low mass haloes are partially broken up by high mass haloes than the inverse. Similar results are found in the $N$-body simulations with different values of the metric parameters $\Omega_{0}$ and $\lambda_{0}$ 


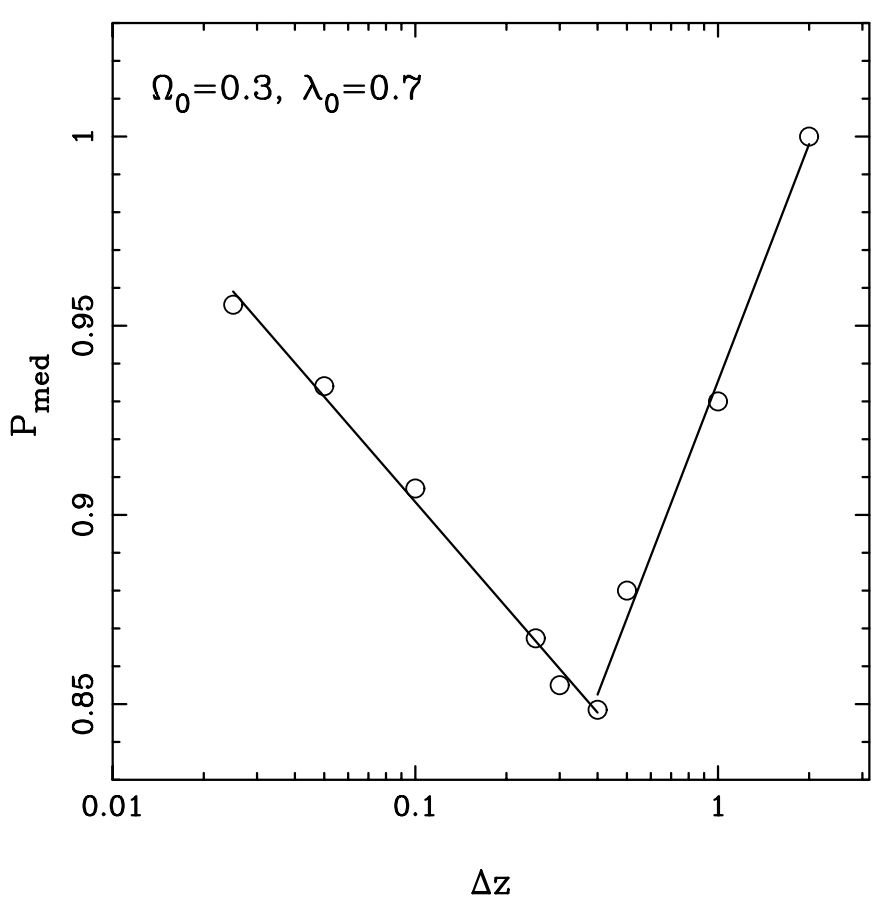

Fig. 2. Dependence of the median fraction $P_{\text {med }}$ of a progenitor halo present in a successor halo as a function of $N$ body simulation output time redshift interval $\Delta z$, for the $\left(\Omega_{0}=0.3, \lambda_{0}=0.7\right)$ CDM-like simulation detailed in Sect. 2.3, in the redshift range $1 \geq z \geq 0.5$ for $\Delta z<0.5$ and $6 \geq z \geq 0$ for $\Delta z \geq 0.5$. Each value obtained from a set of simulation output times is shown as a circle. Linear fits to $P_{\text {med }}$ as a function of $\log _{10}(\Delta z)$ above and below the $\Delta z=0.4$ point are shown here and given in Eq. (5).

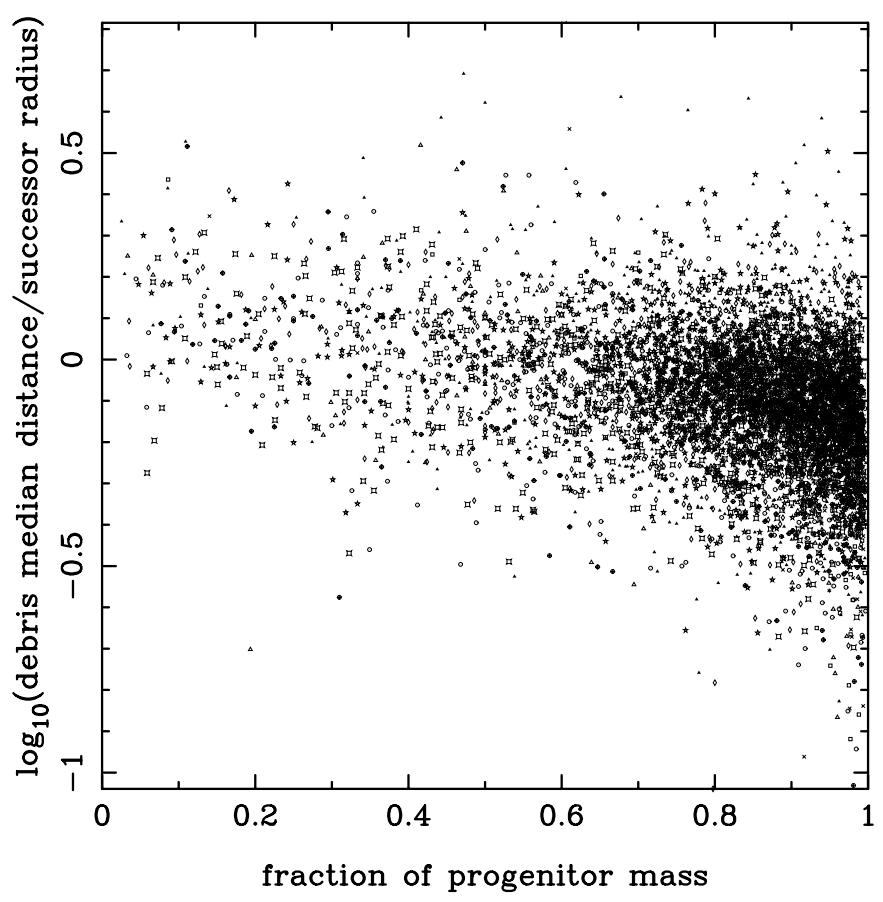

Fig. 3. The ratio of the median distance of debris to the outermost radius of a successor halo, as a function of the fraction of a progenitor present in the successor, for the simulation used in Fig. 1. Symbols are as for Fig. 1.
Table 2. Median merging fractions $P$ as a function of redshift $z_{i}$ and of redshift interval $\Delta z$ between $N$-body simulation output times at $z_{i}$ and $z_{i}-\Delta z$, for the simulations of metric parameters indicated. Note that although the merging fractions for the two low density models are identical to two decimal places, several values differ at the third decimal place (not quoted).

\begin{tabular}{lccccc}
\hline$\left(\Omega_{0}, \lambda_{0}\right)=(1.0,0.0)$ & $(0.3,0.7)$ & $(0.3,0.7)$ & $(0.3,0.7)$ & $(0.3,0.0)$ \\
$z_{i}$ & $\Delta z=0.5$ & $\Delta z=0.5$ & $\Delta z=1$ & $\Delta z=2$ & $\Delta z=0.5$ \\
\hline 6 & 1.00 & 1.00 & 1.00 & 1.00 & 1.00 \\
5.5 & 1.00 & 1.00 & & & 1.00 \\
5 & 1.00 & 1.00 & 1.00 & & 1.00 \\
4.5 & 1.00 & 0.98 & & & 0.98 \\
4 & 0.99 & 0.97 & 0.96 & 1.00 & 0.97 \\
3.5 & 0.97 & 0.94 & & & 0.94 \\
3 & 0.93 & 0.90 & 0.89 & & 0.90 \\
2.5 & 0.90 & 0.87 & & & 0.87 \\
2 & 0.86 & 0.86 & 0.90 & 1.00 & 0.86 \\
1.5 & 0.86 & 0.85 & & & 0.85 \\
1 & 0.84 & 0.85 & 0.95 & & 0.85 \\
0.5 & 0.88 & 0.88 & & & 0.88 \\
\hline overall medians & 0.89 & 0.88 & 0.93 & 1.00 & 0.88 \\
\hline \multicolumn{7}{r}{} & \multicolumn{5}{c}{}
\end{tabular}

Table 3. Dependence on minimum number of particles per halo of median merging fractions $P$, as a function of redshift $z_{i}$ between $N$-body simulation output times at $z_{i}$ and $z_{i}-0.5$, for the $\left(\Omega_{0}, \lambda_{0}\right)=(0.3,0.7)$ simulation. (The column for $N_{\min }=30$ is identical to that in Table 2).

\begin{tabular}{lccc}
\hline$z_{i}$ & $N_{\min }=10$ & $N_{\min }=30$ & $N_{\min }=100$ \\
\hline 6 & 1.00 & 1.00 & 0.99 \\
5.5 & 1.00 & 1.00 & 0.99 \\
5 & 1.00 & 1.00 & 0.99 \\
4.5 & 1.00 & 0.98 & 0.97 \\
4 & 0.95 & 0.97 & 0.95 \\
3.5 & 0.90 & 0.94 & 0.93 \\
3 & 0.82 & 0.90 & 0.92 \\
2.5 & 0.73 & 0.87 & 0.91 \\
2 & 0.67 & 0.86 & 0.90 \\
1.5 & 0.67 & 0.85 & 0.88 \\
1 & 0.70 & 0.85 & 0.87 \\
0.5 & 0.80 & 0.88 & 0.87 \\
\hline \multicolumn{3}{l}{ overall medians } \\
\multicolumn{3}{l}{} \\
\hline
\end{tabular}

(and also in simulations with different initial perturbation spectra). In the present case, a conservative minimum of $N_{\text {min }}=30$ particles per halo was used in application of the friends-of-friends (FOF) group finding algorithm, so this is unlikely to be an effect of resolution.

Nevertheless, the merging percentages of many haloes more massive than $10^{12} M_{\odot}$ are well below $2 P_{\text {med }}-1$, where $P_{\text {med }} \approx 90 \%$ from Table 2 , i.e. well below $80 \%$. 
This confirms the utility of studying the possible effects of the break-up of these haloes on star formation.

Could it be useful to consider analyses with smaller or larger minimum numbers of particles per halo? While the calculation of Poisson errors of the statistics of interest here would not be simple, these can nevertheless be discussed at an order of magnitude level.

The Poisson uncertainty in a halo of $N_{\min }=$ 30 particles is $\sqrt{30}$, i.e. a fractional error of $18 \%$. This is of similar order of magnitude to the median losses represented in Fig. 1 and Table 2.

However, most haloes have masses considerably greater than the minimum required, so while uncertainties due to Poisson noise at the low mass cutoff may be important for the smallest haloes, it is unlikely to be globally important. So, increasing the cutoff criterion to $N_{\text {min }}=100$ particles should not greatly affect the analysis. Table 3 confirms that median merging fractions are affected only slightly.

Table 4 (Sect. 3.1.3) shows that more subtle statistics, such as relative orientations of debris and non-debris particles' vectors to successor halo centres, are modified somewhat more, particularly at earlier redshifts. This is to be expected, since at early redshifts, high mass haloes do not yet exist, so more haloes are closer to the cutoff limit at high redshift than at low redshift.

On the other hand, decreasing the cutoff, to $N_{\min }=$ 10 particles, for example, is certainly going to increase Poisson noise: $33 \%$ is a high uncertainty even for estimates of first order statistics.

Table 3 supports this argument, showing that lowering from $N_{\min }=30$ to $N_{\min }=10$ has considerably greater effect (at $z \leq 3)$ than that of increasing to $N_{\min }=100$.

Therefore, the criterion of $N_{\text {min }}=30$ appears to be within the region of convergence of the most basic debris statistic as a function of $N_{\min }$. The more subtle statistics shown in Table 4 support $N_{\min }=30$ as being closer to a domain of convergence than $N_{\min }=10$.

Hence, results below are all for $N_{\min }=30$ unless otherwise specified.

\subsection{2. (ii) How do the debris fractions depend on the output time (redshift) and the output time (redshift) intervals?}

Table 2 shows that, in general, the median merging percentages start close to $100 \%$ and decrease as time increases. It was remarked upon above that the break-up of low mass haloes by high mass haloes is more physically reasonable than the opposite. If this is a necessary condition for the break-up of haloes, then it is necessary to have a large dynamic range in halo masses in order for the mass ratios to be large enough for the effect to occur.

For a given $N$-body simulation in which halo formation is (bottom-up) hierarchical, and which has a fixed mass resolution independently of time, the first haloes to collapse necessarily have roughly equal masses, just slightly greater than the detection threshold. It is only at later times that high mass and low mass haloes can coexist, and so, as is suggested here, the high mass haloes can tend to cause the low mass haloes to "lose" some of their mass outside of halo boundaries defined by a given groupfinding criterion.

This provides one possible explanation for why the median merging fractions can decrease with time as shown in Table 2.

An alternative explanation can be expressed in terms of dynamical times. The fraction of debris loss $1-P$ should be related in some way to the fraction of a dynamical time represented by an interval $\Delta z$. Since a fixed (virialisation) overdensity threshold is used to detect haloes,

$t_{\text {dyn }} \propto \rho_{\text {halo }}^{-1 / 2} \propto\langle\rho\rangle^{-1 / 2} \propto(1+z)^{-3 / 2}$,

so that

$\frac{\mathrm{d} t_{\mathrm{dyn}}}{t_{\mathrm{dyn}}} \propto \frac{\mathrm{d} z}{(1+z)}$

Hence, a fixed choice of interval $\Delta z$ corresponds to fractions of a dynamical time $\mathrm{d} t_{\mathrm{dyn}} / t_{\mathrm{dyn}}$ which increase with increasing time (decreasing $z$ ).

The interpretation of the time dependence of $P_{\mathrm{med}}$ in Table 2 is therefore that at early times, $\Delta z$ corresponds to dynamical time fractions that are too short to have time for much debris loss. As $\mathrm{d} t_{\mathrm{dyn}} / t_{\mathrm{dyn}}$ increases, the loss fractions increase $\left(P_{\text {med }}\right.$ decreases $)$, until $\mathrm{d} t_{\mathrm{dyn}} / t_{\mathrm{dyn}}$ becomes large enough that matter has time to fall back into the haloes, in which case the loss fraction decreases ( $P_{\text {med }}$ increases).

The latter effect is also shown by the dependence on redshift interval revealed in Table 2 . This shows that increasing the time interval (at constant redshift) enables the debris from progenitor haloes to mostly fall into the successor haloes. This implies that although the problem of debris may temporarily modify the monotonic, spherically symmetric infall assumption, the effect is limited in time.

Table 2 also shows that increasing the time interval (redshift interval) shifts the minimum in $P_{\text {med }}$ to earlier times. This is consistent with the above explanation.

If the redshift interval is made shorter than the values shown in Table 2, then the time resolution becomes smaller than typical dynamical times, so that debris does not have time to be lost between successive $N$-body output times. Between the two extremes of small intervals over which debris does not have time to be ejected, and large intervals over which there is sufficient time for debris to fall back into the successor haloes, a minimum in $P_{\text {med }}$ (a maximum debris loss) must occur.

Figure 2 shows this for the $\left(\Omega_{0}=0.3, \lambda_{0}=0.7\right)$ case. The maximum debris loss occurs at $\Delta z \approx 0.4$, and the two dependences of debris loss above and below the minimum can be estimated as:

$P_{\text {med }} \approx\left\{\begin{array}{l}81 \%-9 \% \log _{10}(\Delta z), \quad 0.025 \lesssim \Delta z \lesssim 0.4 \\ 94 \%+21 \% \log _{10}(\Delta z), \quad 0.4 \lesssim \Delta z \lesssim 2\end{array}\right.$ 


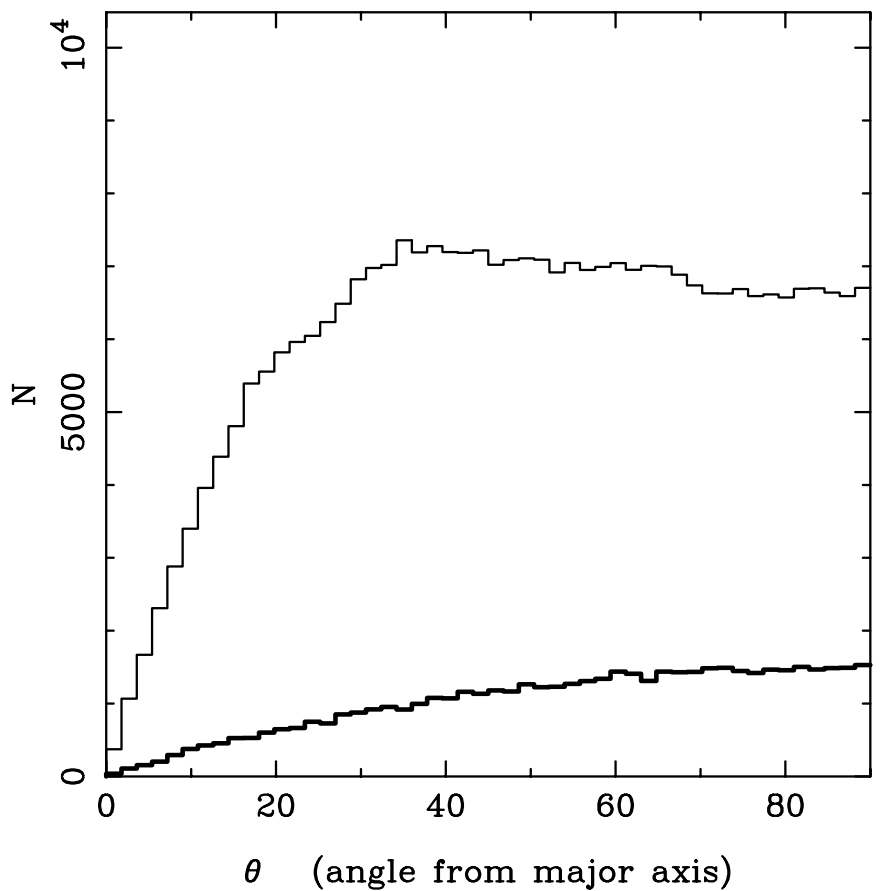

Fig. 4. Distributions of angles $\theta$ (in degrees) between (i) the vector from the centre of a successor halo to either a halo (nondebris) particle or a debris particle and (ii) the major axis of the successor halo, for the $z \in[1.5,1.0]$ interval of the $\left(\Omega_{0}=\right.$ $0.3, \lambda_{0}=0.7$ ) simulation (Sect. 3.1.3). The thin curve is for non-debris particles, the thick curve is for debris particles. The non-debris vectors are clearly more aligned with the major axis. An isotropic distribution would follow a $\sin \theta$ function.

Table 4. Mean angles $\theta$ (defined in caption of Fig. 4) for non-debris halo $(\mathrm{H})$ and debris $(\mathrm{D})$ particles, their difference $\delta \theta$, and the $1 \sigma$ uncertainty in the difference $\Delta(\delta \theta)$ [the uncertainty $\Delta(\delta \theta)$ is estimated by using the standard deviations of $\theta$, taking standard errors in the mean, and assuming Gaussian error propagation], as a function of redshift $z_{i}$, between $N$-body simulation output times at $z_{i}$ and $z_{i}-0.5$, for the $\left(\Omega_{0}, \lambda_{0}\right)=(0.3,0.7)$ simulation, for $N_{\min }=30$ by default, and for other values of $N_{\min }$ as indicated. The means are weighted by $\sin \theta$ and angles $\theta<5^{\circ}$ are ignored. Units are degrees.

\begin{tabular}{|c|c|c|c|c|c|}
\hline \multirow[b]{2}{*}{$z_{i}$} & \multicolumn{2}{|c|}{$N_{\min }=30$} & \multirow[t]{2}{*}{$N_{\min }=10$} & \multirow{2}{*}{$\begin{array}{l}N_{\min }=30 \\
\delta \theta \pm \Delta(\delta \theta)\end{array}$} & \multirow{2}{*}{$N_{\min }=100$} \\
\hline & $\mathrm{H}$ & $\mathrm{D}$ & & & \\
\hline 6.0 & 25.08 & 24.25 & $0.8 \pm 1.6$ & $-0.8 \pm 1.8$ & $-7.9 \pm 1.6$ \\
\hline 5.5 & 25.02 & 15.31 & $-3.3 \pm 1.1$ & $-9.7 \pm 1.2$ & $-13.5 \pm 1.4$ \\
\hline 5.0 & 25.68 & 19.87 & $-0.4 \pm 0.8$ & $-5.8 \pm 0.9$ & $-11.2 \pm 1.0$ \\
\hline 4.5 & 25.99 & 22.75 & $-0.1 \pm 0.4$ & $-3.2 \pm 0.5$ & $-5.5 \pm 0.6$ \\
\hline 4.0 & 31.14 & 23.13 & $-4.5 \pm 0.3$ & $-8.0 \pm 0.3$ & $-11.0 \pm 0.3$ \\
\hline 3.5 & 33.79 & 38.97 & $6.5 \pm 0.2$ & $5.2 \pm 0.3$ & $7.3 \pm 0.3$ \\
\hline 3.0 & 34.20 & 35.62 & $3.7 \pm 0.2$ & $1.4 \pm 0.2$ & $0.1 \pm 0.2$ \\
\hline 2.5 & 39.05 & 43.22 & $5.1 \pm 0.1$ & $4.2 \pm 0.2$ & $4.1 \pm 0.2$ \\
\hline 2.0 & 37.43 & 45.18 & $7.5 \pm 0.1$ & $7.8 \pm 0.1$ & $9.5 \pm 0.2$ \\
\hline 1.5 & 38.12 & 45.20 & $7.2 \pm 0.1$ & $7.1 \pm 0.1$ & $7.3 \pm 0.1$ \\
\hline 1.0 & 36.76 & 42.42 & $5.9 \pm 0.1$ & $5.7 \pm 0.1$ & $5.6 \pm 0.1$ \\
\hline 0.5 & 36.97 & 42.22 & $5.3 \pm 0.1$ & $5.3 \pm 0.1$ & $5.1 \pm 0.1$ \\
\hline
\end{tabular}

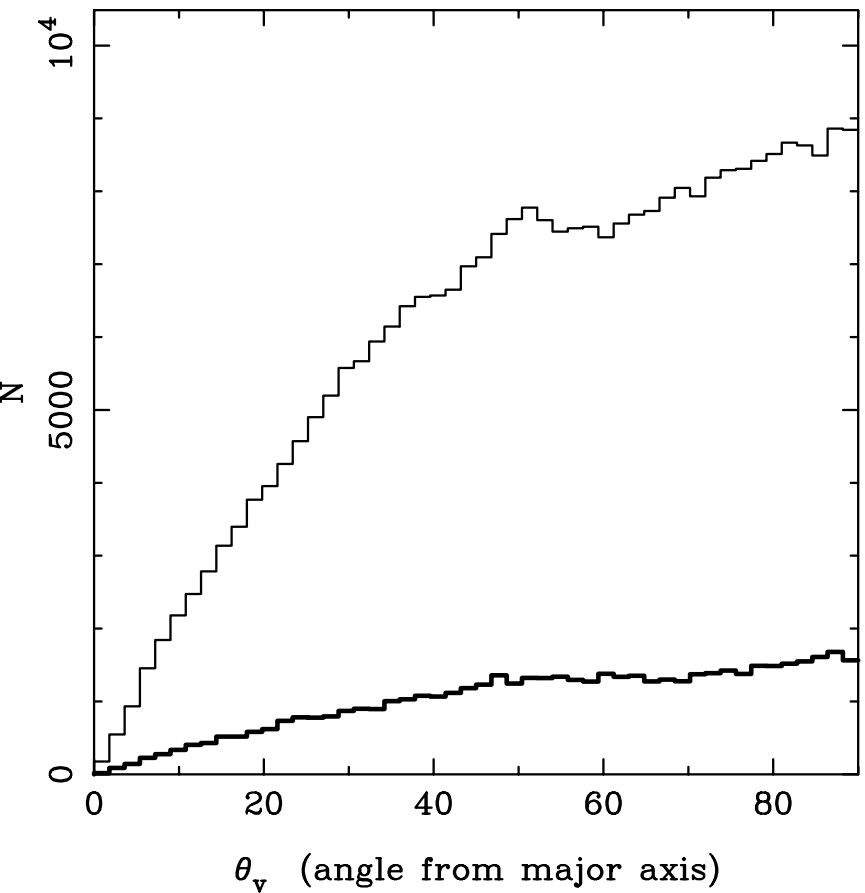

Fig. 5. As for Fig. 4, except that distributions of angles $\theta_{\mathrm{V}}$ (in degrees) between debris and non-debris particles' velocity vectors and successor halo (spatial) major axes, using the successor halo velocity and spatial centres as origins, are shown. As for Fig. 4, the thin curve is for non-debris particles, the thick curve is for debris particles. The two distributions are not as strikingly different as those in Fig. 4.

though clearly these relations can only hold over approximately the intervals stated, since for small intervals, $P_{\text {med }} \rightarrow 1^{-}$as $\Delta z \rightarrow 0^{+}$and for large intervals, $P_{\text {med }} \leq 1$ implies that the relation cannot continue above $P_{\text {med }}=1$.

It should also be noted that the formal uncertainties on $P_{\text {med }}$ diminish as $P_{\text {med }} \rightarrow 1$, since if the number of particles in a progenitor halo is $N$, then the binomial uncertainty in $N P_{\text {med }}$ is $\sqrt{N P_{\text {med }}\left(1-P_{\text {med }}\right)}$, so that

$$
\begin{aligned}
\Delta P_{\text {med }} & =\Delta\left[\left(N P_{\text {med }}\right) / N\right] \\
& =\Delta\left[N P_{\text {med }}\right] / N \\
& =\sqrt{N P_{\text {med }}\left(1-P_{\text {med }}\right)} / N \\
& =\sqrt{P_{\text {med }}\left(1-P_{\text {med }}\right)} / \sqrt{N} \\
& \rightarrow 0 \text { as } P_{\text {med }} \rightarrow 1 .
\end{aligned}
$$

Comparison with Table 3 of Roukema et al. (1997) (which shows mean values of $P$, for less conventional choices of group-finding detection thresholds) indicates that the various $P$ estimates will vary somewhat with choice of groupfinder and initial perturbation spectrum.

However, presumably because Eq. (5) is based on a redshift interval rather than a time interval, it appears nearly independent of the metric parameters $\Omega_{0}$ and $\lambda_{0}$. 
Table 5. Mean velocity angles $\theta_{\mathrm{V}}$ (as in Fig. 5) for non-debris halo $(\mathrm{H})$ and debris $(\mathrm{D})$ particles, their difference $\delta \theta_{\mathrm{v}}$, and the $1 \sigma$ uncertainty in the difference $\Delta\left(\delta \theta_{\mathrm{V}}\right)$ [the uncertainty $\Delta\left(\delta \theta_{\mathrm{v}}\right)$ is estimated by using the standard deviations of $\theta$, taking standard errors in the mean, and assuming Gaussian error propagation], as a function of redshift $z_{i}$, between $N$-body simulation output times at $z_{i}$ and $z_{i}-0.5$, for the $\left(\Omega_{0}, \lambda_{0}\right)=$ $(0.3,0.7)$ simulation, for $N_{\text {min }}=30$. The means are weighted by $\sin \theta$ and angles $\theta<5^{\circ}$ are ignored. Units are degrees.

\begin{tabular}{lccc}
\hline$z_{i}$ & $\mathrm{H}$ & $\mathrm{D}$ & $\delta \theta_{\mathrm{v}} \pm \Delta\left(\delta \theta_{\mathrm{v}}\right)$ \\
\hline 6.0 & 50.13 & 52.42 & $2.3 \pm 2.4$ \\
5.5 & 50.87 & 47.40 & $-3.5 \pm 2.2$ \\
5.0 & 51.50 & 46.08 & $-5.4 \pm 1.0$ \\
4.5 & 50.28 & 48.36 & $-1.9 \pm 0.5$ \\
4.0 & 47.66 & 49.59 & $1.9 \pm 0.3$ \\
3.5 & 49.61 & 49.59 & $0.0 \pm 0.3$ \\
3.0 & 47.92 & 46.98 & $-0.9 \pm 0.2$ \\
2.5 & 46.79 & 47.39 & $0.6 \pm 0.2$ \\
2.0 & 47.67 & 47.17 & $-0.5 \pm 0.1$ \\
1.5 & 44.23 & 45.40 & $1.2 \pm 0.1$ \\
1.0 & 44.24 & 44.62 & $0.4 \pm 0.1$ \\
0.5 & 45.54 & 45.73 & $0.2 \pm 0.1$ \\
\hline
\end{tabular}

\subsection{3. (iii) How far from the central halo are debris located?}

Figure 3 shows that, in general, the debris is located close to the successor haloes. This is reassuring for those who wish to ignore the debris. In fact, the median distances of particle debris are mostly closer to the centre of the successor halo than its outermost radius. This would not be possible if a spherical overdensity group-finder were used, but since dark matter haloes are generally found to be triaxial (in the sense that they are not, in general, spherically symmetric, e.g. Warren et al. 1992; Cole \& Lacey 1996), this simply means that the vectors from debris to the successor halo centre are less aligned with the major axis of the successor halo than are the vectors from non-debris halo particles. This suggests that the debris are also more likely to be in orbit around one (or both) of the minor axes of the successor halo than non-debris particles.

These explanations are examined quantitatively at the epoch of largest debris loss in Figs. 4 and 5, and as a function of time in Tables 4 and 5 .

Overall, these figures and tables clearly show that the debris particles' position vector distributions have significantly different spatial orientations to those of the nondebris particles by several degrees, relative to the halo major axes. Given the formal errors listed, some of the velocity distributions would also appear to have significant differences, but the lack of a smooth trend suggests that this may just be due to noise.

So, while the spatial alignment of debris relative to non-debris is confirmed to avoid the major axes, as was suggested above to explain Fig. 3, kinematic differences in the two populations appear to be very small.

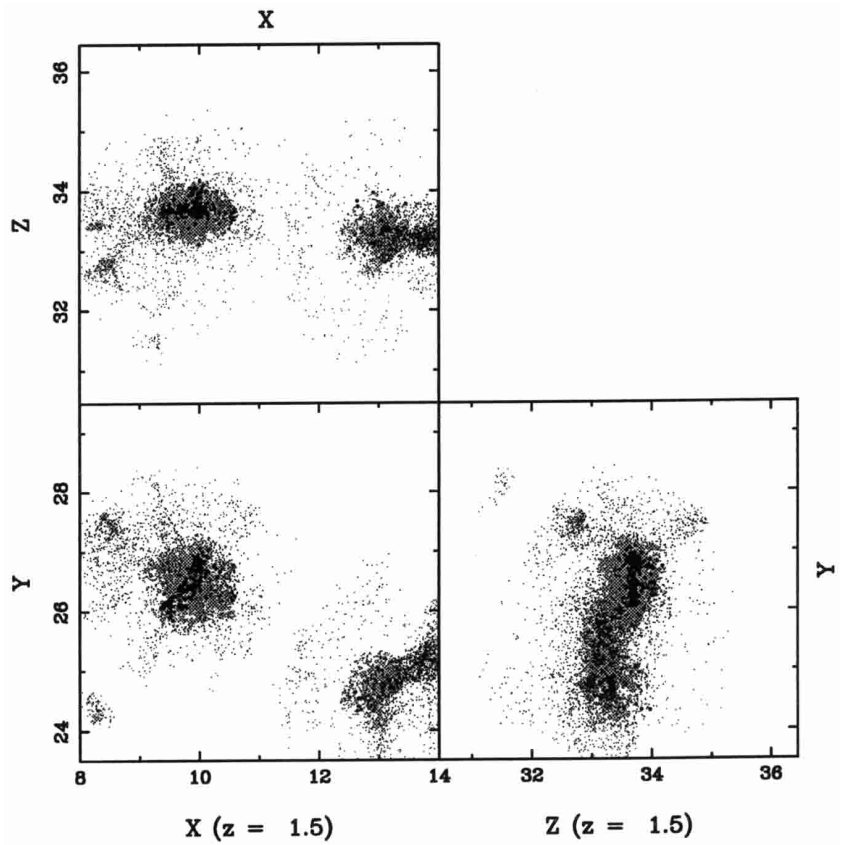

Fig. 6. Example of debris in a $2.6 h^{-1} \mathrm{Mpc}$ cube around an interesting halo, shown in this figure and in Figs. 7 and 8. Dark matter which, at $z=1.0$, is either detected in a (primary) successor or newly formed halo (small black points, "virialised" matter), detected in a secondary successor "halo" (large black points, i.e. "re-virialised" debris), or which constitutes ordinary debris (large gray points) is shown at the pair of output times used to determine these criteria: $z=1.5$ is the first output time (this figure), $z=1.0$ is the second output time (Fig. 7). Background particles are not shown. The point styles/shadings of any given particle are physically determined at $z=1.0$ and kept identical for the earlier image at $z=1.5$ and for the later image at $z=0.5$. Axes are labelled in comoving Mpc. Projections are rectilinear $X-Y-Z$ as indicated. In this image $(z=1.5)$, all the particles destined to become debris, shown by large dots, clearly occupy the dense bodies of the two big haloes. Many particles destined to fall into the haloes, i.e. destined to satisfy the group-finder criterion at the following output time, are also visible as scatterings of small dots around the haloes.

Moreover, Table 4 quantitatively shows the shift from mostly filamentary structures at high redshifts to much more symmetric structures at later times. It also shows that the debris orientations are closer to the major axes at earlier times. This filamentarity starts from around $10^{\circ}$ more "filamentarity" at $z_{i}=5.5$ (ignoring $\theta$ for the $z \in[6.0,5.5]$ interval, which is insignificantly different from zero), and decreases and switches through to a maximum debris "oblateness" approximately coinciding with the epochs of maximum debris loss at the same time as the non-debris material becomes more spherically symmetric.

Another property revealed by Fig. 3 is that the general slope of the relation in the figure shows that the higher the fraction of a progenitor halo is expelled as debris (i.e. the lower the merging fraction), the more likely this debris is to be further from the centre of the successor halo. 
$\mathrm{x}$

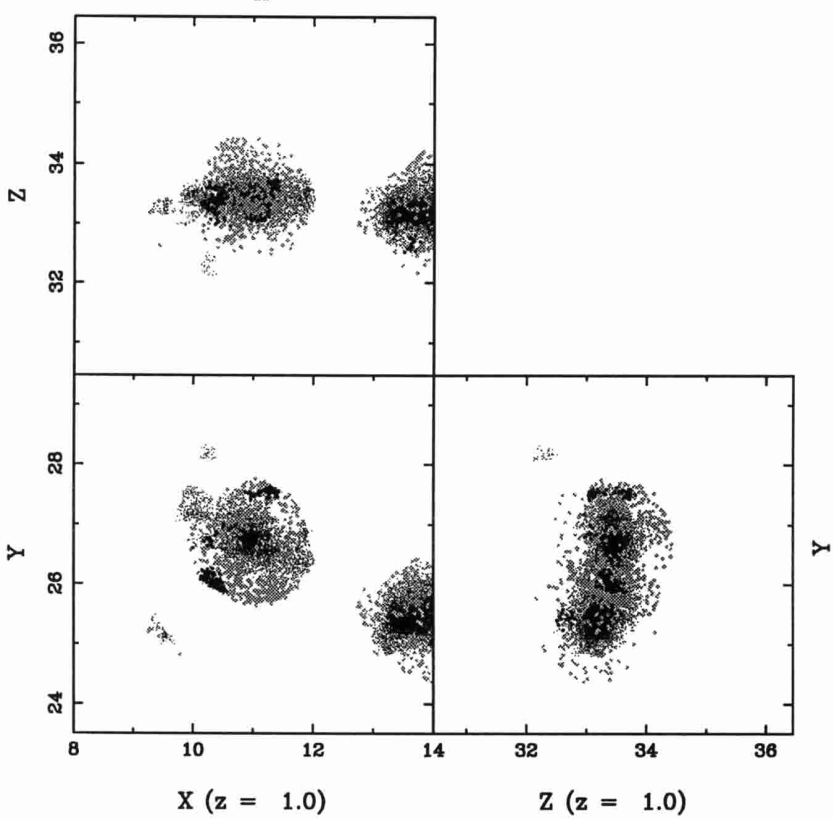

Fig. 7. As for Fig. 6, for the output time at which classification into the three particle types shown is carried out (background particles are ignored), i.e. at $z=1.0$. Two small "re-virialised" shells (large black dots) are visible in the $X-Y$ image around the main halo: at the bottom-left and at the top of this halo, i.e. mainly separated in the $Y$ direction, with a small separation in the $X$ direction. A possible shell/tail is visible for the higher $X$ value main halo. The former halo is moving towards greater $X$ values and will merge with the latter, as it is falling along a large filament towards a deep potential well (not shown here).

Table 6. Differing fates at an output time $t_{i+1}$ of matter which is "virialised" at output time $t_{i}$ : inclusion in a primary successor or newly collapsed halo $(\mathrm{H})$, non-virialised debris $(\mathrm{NV})$, or re-virialised (RV, secondary successor halo) debris (any particle can only be a member of one category), and the fraction $f \equiv N(\mathrm{RV}) /[N(\mathrm{NV})+N(\mathrm{RV})]$ of debris which is "re-virialised", as a function of redshift $z_{i}$, between $N$-body simulation output times at $z_{i}$ and $z_{i}-0.5$, for the $\left(\Omega_{0}, \lambda_{0}\right)=$ $(0.3,0.7)$ simulation. The total number of particles is $N=$ 2097152 and the largest successor criterion is used as the merger/identity criterion in order to maximise tracing of debris.

\begin{tabular}{lcccc}
\hline$z_{i}$ & $\mathrm{H}$ & $\mathrm{NV}$ & $\mathrm{RV}$ & $f$ \\
\hline 6 & 7135 & 93 & 0 & 0 \\
5.5 & 14648 & 100 & 40 & 0.4 \\
5 & 28032 & 383 & 110 & 0.3 \\
4.5 & 47191 & 1444 & 755 & 0.52 \\
4 & 76986 & 4032 & 1785 & 0.44 \\
3.5 & 120674 & 8562 & 2006 & 0.23 \\
3 & 178627 & 15102 & 2346 & 0.16 \\
2.5 & 252710 & 23503 & 2456 & 0.10 \\
2 & 354118 & 30839 & 2651 & 0.09 \\
1.5 & 478918 & 46726 & 4440 & 0.10 \\
1 & 632984 & 61960 & 6045 & 0.10 \\
0.5 & 833417 & 61038 & 5618 & 0.09 \\
\hline
\end{tabular}

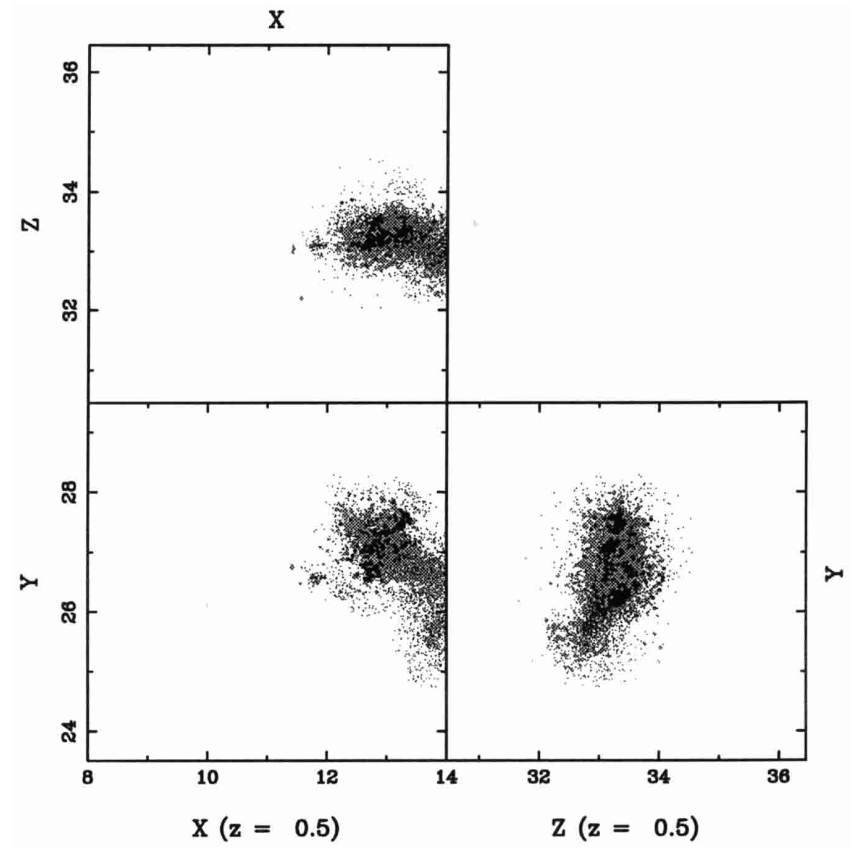

Fig. 8. As for Figs. 6 and 7, for a later output time, i.e. at $z=0.5$. Both the "re-virialised" shells (large black dots) visible in the $X-Y$ view of the halo at $z=1.0$ in Fig. 7 and the ordinary debris (large gray dots) around the same halo have clearly fallen in to the main body of the halo, which has moved further towards greater $X$ values to start merging with its neighbour.

This is dynamically unsurprising: the less bound the progenitor is to the successor, the lower the merging fraction is likely to be and the further matter is likely to be ejected from the successor.

While these subtleties of tidal debris dynamics are unlikely to have significant effects on global star formation statistics at the broad level studied in this initial exploration of tidal debris effects, these results could presumably be taken into account in future second order studies.

\subsection{4. (iv) Does the debris just form loose atmospheres and tidal tails, or does it revirialise and form tidal dwarf haloes/galaxies?}

Figures 6, 7 and 8 show an example of material classified as debris in one of the $N$-body simulations studied, using the largest successor criterion in order to maximise the number of particles accounted for as debris.

Quantitatively, material which is merely low density is distinguished from material which has "re-virialised", i.e. which is detected as part of a secondary halo at the later output time ( $z=1.0$ in the case illustrated). The former is traced by large gray dots and the latter by large black dots.

Qualitative distinctions in the different forms of debris can be made by visual inspection of the figures. The low density material (large gray dots) appears to form a loose atmosphere, while the "re-virialised" matter appears 
to form several temporary "shells" (bunches of large black dots at the bottom left and at the top of the $X-Y$ view of the main halo in Fig. 7, i.e. at $z=1.0$ ). Shells are known to form as a general consequence of merging bodies under gravitation, and have been observed in optical images of galaxies (Malin et al. 1983; Quinn 1984; Hernquist \& Quinn 1988, 1989).

In the latter category, is it useful to try to distinguish genuinely re-virialised matter, i.e. tidal dwarf haloes which are bound objects, from the temporary phenomena of tidal tails and shells which recollapse to the main halo?

The analyses of Dubinski et al. (1996), Mihos et al. (1998) and Springel \& White (1999) suggest that whether or not tidal tail dwarf haloes/galaxies can be formed in models of merging gravitational discs depends on the details of the galaxy formation recipe adopted.

Since dissipation is not included in the pure gravity, $N=2 \times 10^{6}$-body simulations studied here, realistic discs are unlikely to form and robust constraints on virialised tidal dwarfs cannot be expected.

Moreover, observations (Duc \& Mirabel 1994; Duc et al. 1997; Mendes de Oliveira et al. 2000) imply masses of tidal tail galaxies of around $10^{9}-10^{10} M_{\odot}$, a scale which is well below that of masses corresponding to $L^{*}$, the characteristic 'shoulder' mass of the galaxy luminosity function, of around $10^{11}-10^{12} M_{\odot}$. These tidal dwarf masses are also below the 30-particle minimum mass of haloes detected in the present simulations: $6 \times 10^{10} M_{\odot}$ (for the $\Omega_{0}=0.3$ simulations, where particle mass is $2 \times 10^{9} M_{\odot}$ ).

In any case, the amount of "re-virialised" matter, whether in unbound tidal tails and shells or in bound tidal dwarfs, is small. This result is shown in Table 6 , for the $\Delta z=0.5$ interval, which gives close to maximum debris fractions.

Comparison of Tables 2 and 6 shows that once the amount of debris becomes non-negligible, i.e. $10 \%$ or greater (during the $z \in[3.0,2.5]$ interval for the nonzero cosmological constant metric), the fraction of this debris which is comprised of re-virialised matter approaches around $10 \%$. That is, the fraction of virialised matter at one time step which does not contribute to the most massive successor halo of a given halo, but instead to a secondary "halo", is only around $\sim 1 \%$ or less.

This is consistent with the expectations from observational limits on tidal dwarf formation, and suggests that tidal dwarfs are unlikely to contribute significantly to first order global galaxy statistics, even if all tidal tails and shells which passed through dense stages were considered to form independent, bound systems.

\subsection{Mass functions}

Figure 9 shows that use of the stricter merger/identity criterion, i.e. the $P=78 \%$ median criterion instead of the largest successor criterion, causes a strong effect in the low mass haloes and little (global) effect in the high mass haloes. This is consistent with what can be expected from

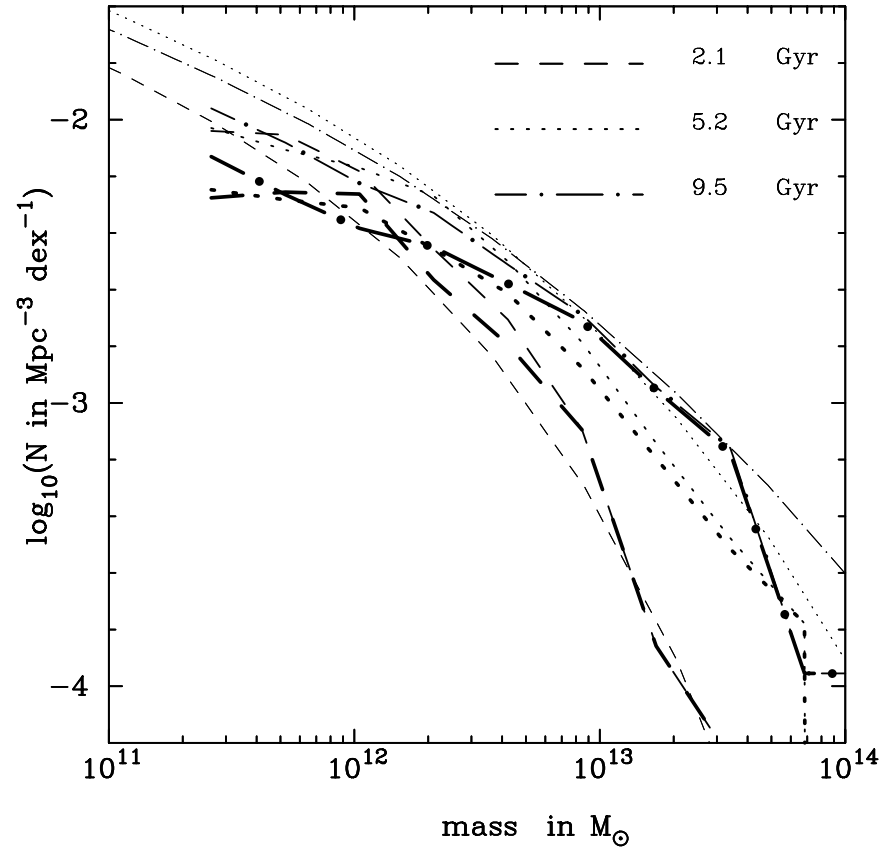

Fig. 9. Halo mass function evolution (thick lines) based on the merger history tree interpolated between the $N$-body simulation outputs referred to in Fig. 1. The very thin curves indicate Press-Schechter mass functions for the curvature and perturbation spectrum parameters indicated above, where a top-hat window function, $\delta_{c 0}=1.3$ and a short wavelength smooth truncation at $1 / 64$ of the box size are applied. The medium thickness curves are for the largest successor merger/identity criterion (Sect. 2.2), i.e. including debris, and the very thick curves are for the median merger/identity criterion (Sect. 2.1), where $P \equiv 2 P_{\text {med }}-1=78 \%$, i.e. excluding a significant fraction of debris. The median criterion mass functions are slightly lower than the largest successor criterion mass functions at the low mass end.

Fig. 1, since the haloes which have less than $P=78 \%$ merging fractions are mostly, but not all, of small masses.

Note that these mass functions are constructed from output times independent of the original $N$-body simulation output times. Sets of haloes are detected in individual $N$-body output times, a halo merging history tree is then constructed, a galaxy+halo merging history tree is calculated, and finally a star formation rate history tree is evaluated. The mass functions shown represent the parent haloes of all galaxies present in the star formation history tree at the chosen (arbitrary) output time which existed as individual haloes at the $N$-body simulation output time $t_{i}$ preceding the chosen output time. This provides a double check on the interpolation and merging time algorithms, since the set of haloes represented here in a mass function should be a subset of the haloes present at $t_{i}$ and a superset of the haloes present at $t_{i+1}$.

With the application of the median merger/identity criterion, it is often the case that a halo at a time $t_{i}$ has no successor at time $t_{i+1}$ because of the strictness of the criterion $\left(P>2 P_{\text {med }}-1\right.$ for the median criterion compared to $P>0$ for the largest successor criterion). In this case, 


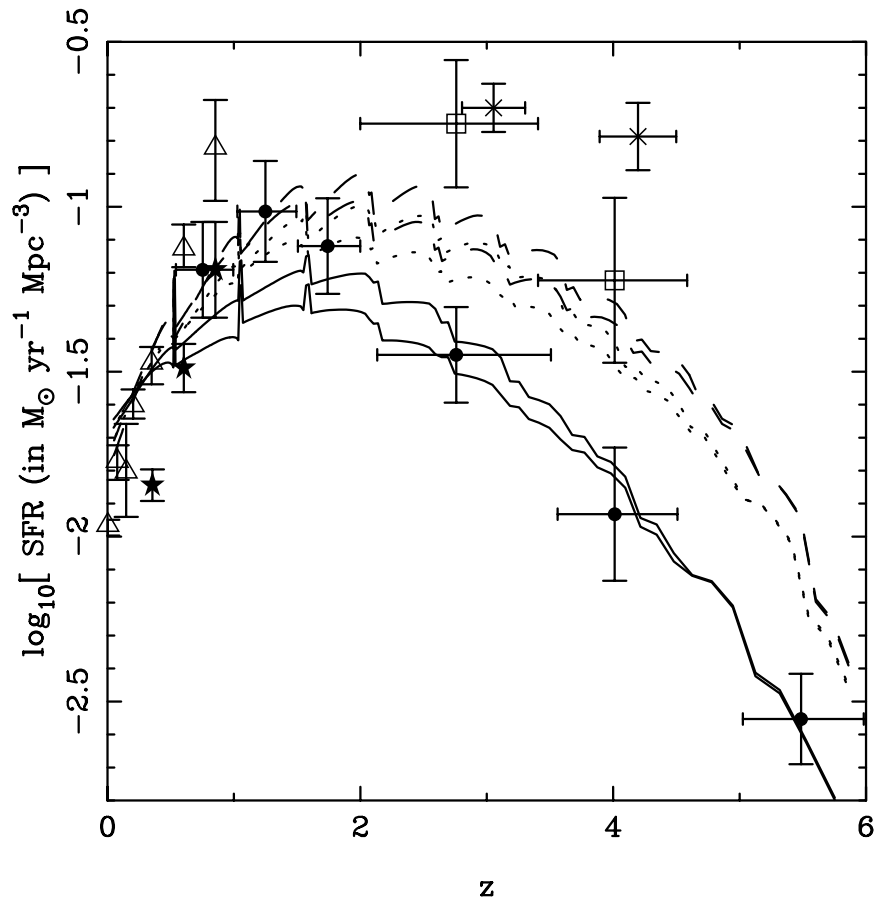

Fig. 10. Global star formation rate histories for the "largest successor" merger/identity criterion (upper curve of each pair) and the "median" criterion (lower curve of each pair). The solid, dashed and dotted curves are for the models with $\left(\Omega_{0}, \lambda_{0}\right)=(1.0,0.0),(0.3,0.7)$ and $(0.3,0.0)$ respectively. Observational points (taken from summary in Fig. 9 of Blain et al. 1999) are shown as open triangles (Gallego et al. 1996; Gronwall 1999; Treyer et al. 1998; Tresse \& Maddox 1998; Hammer \& Flores 1999), filled stars (Lilly et al. 1996), filled circles (Connolly et al. 1997; Madau et al. 1996), open squares (dust-corrected, Pettini et al. 1998) and " $\times$ " crosses (dustcorrected, Steidel et al. 1999). All models and observational estimates are converted to $\left(\Omega_{0}, \lambda_{0}, h\right)=(1.0,0.0,0.65)$ for plotting purposes.

Table 7. Effect of merger/identity criterion choice on global star formation rates (cf. Fig. 10). The decrease in star formation rate due to the exclusion of debris, as represented by using the median rather than the largest successor merger/identity criterion, is $\Delta_{\psi}(z) \equiv$ $\log _{10} \psi(z$, largest successor $)-\log _{10} \psi(z$, median). See Sect. 2.4 for the definitions of $\alpha$ and $\varepsilon$, the efficiency parameters for the star formation rate and reheating respectively.

\begin{tabular}{|c|c|c|c|c|c|c|}
\hline$\Omega_{0}$ & $\lambda_{0}$ & $\alpha$ & $\varepsilon$ & $\Delta_{\psi}(z=1)$ & $\Delta_{\psi}(z=3)$ & $\max \left(\Delta_{\psi}\right)$ \\
\hline \multicolumn{7}{|c|}{ vary metric: } \\
\hline 1.0 & 0.0 & 1.0 & 0.1 & 0.11 & 0.10 & 0.14 \\
\hline 0.3 & 0.7 & 1.0 & 0.1 & 0.04 & 0.11 & 0.12 \\
\hline 0.3 & 0.0 & 1.0 & 0.1 & 0.05 & 0.11 & 0.12 \\
\hline \multicolumn{7}{|c|}{ vary star formation rate: } \\
\hline 0.3 & 0.7 & 0.1 & 0.1 & 0.11 & 0.12 & 0.14 \\
\hline 0.3 & 0.7 & 10.0 & 0.1 & 0.01 & 0.06 & 0.13 \\
\hline \multicolumn{7}{|c|}{ vary gas reheating: } \\
\hline 0.3 & 0.7 & 1.0 & 0.01 & 0.07 & 0.12 & 0.13 \\
\hline 0.3 & 0.7 & 1.0 & 1.0 & 0.00 & 0.06 & 0.09 \\
\hline
\end{tabular}

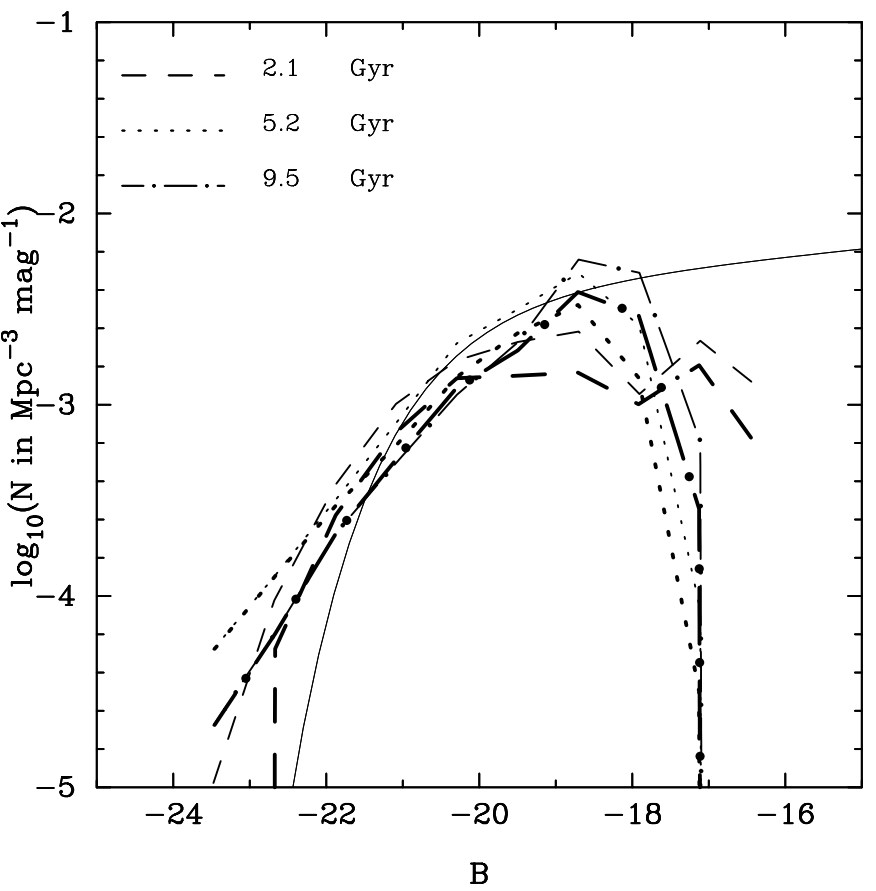

Fig. 11. Luminosity function evolution, as for Fig. 9, medium thickness curves for exclusion of debris, very thick curves for inclusion of debris. The smooth curve to guide the eye is a Loveday et al. (1992) parametrised observational luminosity function.

the halo's mass does not contribute to any branch of the tree during the interval $\left(t_{i}, t_{i+1}\right)$. It may have contributed to the tree during $t<t_{i}$, and some of its constituent total matter particles are likely to contribute to haloes later, during $t>t_{i+1}$, but during the interval $\left(t_{i}, t_{i+1}\right)$ it is considered to be non-existent as far as the star formation rate history tree is concerned.

The physical interpretation of this in terms of mass distribution is that the material from a halo is being "smeared out" as the halo passes through or is torn up by its companion halo(es).

How wrong, in terms of implied star formation or luminosity estimates, is the Press-Schechter approximation in its ignorance of this process? The estimates made here are as follows.

\subsection{Star formation and luminosity functions}

Figures 10 and 11 show how the effect of star formation truncation in haloes which turn into "debris" translates to global star formation rates and luminosity functions.

As discussed in Sects. 2.3 and 2.4, this is carried out using ARFus-V0.03, where adoption of the median (Sect. 2.1) and largest successor (Sect. 2.2) merging/identity criteria test the limiting cases (a) and (b) respectively as defined in Sect. 1 .

The principal effect in the luminosity functions is that the reduction in the number of low mass haloes is visible as a reduction in the number of low luminosity galaxies, and overall as a reduction in the global star formation rate. 
Table 8. Effect of merger/identity criterion choice on differential luminosity functions $\phi(B)$ (shown in Fig. 11 for $\left.\Omega_{0}=1, \lambda_{0}=0\right)$. The decrease in galaxy number density at characteristic absolute magnitudes ("shoulder" and faint end of the luminosity function) due to the exclusion of debris, as represented by using the median rather than the largest successor merger/identity criterion, is $\Delta_{\phi}(B) \equiv$ $\log _{10} \phi(B$, largest successor $)-\log _{10} \phi(B$, median $)$.

\begin{tabular}{cccc}
\hline$\Omega_{0}$ & $\lambda_{0}$ & $\Delta_{\phi}(B=-21)$ & $\Delta_{\phi}(B=-18)$ \\
\hline 1.0 & 0.0 & -0.02 & 0.22 \\
0.3 & 0.7 & 0.03 & 0.16 \\
0.3 & 0.0 & 0.03 & 0.16 \\
\hline
\end{tabular}

Table 8 shows the extent to which the reduction in the number of low luminosity galaxies depends on the metric parameters $\left(\Omega_{0}, \lambda_{0}\right)$. There is some dependence on the matter density, i.e. the reduction in number density is less strong for lower matter density, but there is negligible dependence on the cosmological constant.

The main effect in the global star formation rate (Fig. 10) is a difference of about $0.05-0.10$ dex (see Table 7$)$ at intermediate redshifts $(1 \lesssim z \lesssim 3)$. Given the uncertainty in observational estimates at super-unity redshifts, this is presently an effect smaller than the observational uncertainty.

Table 7 shows that this effect has little dependence on either the metric parameters or on particular choices of the efficiency parameters for the star formation rate and gas reheating. Because of the discreteness in the redshifts of the $N$-body simulation output times, the $\max \left(\Delta_{\phi}\right)$ values are more robust estimates of the effect of debris than the $\Delta_{\phi}(z=1)$ and $\Delta_{\phi}(z=3)$ values, particularly in the cases of $\alpha=10.0$ and $\varepsilon=1$, in which excessively high star formation rates or reheating (respectively) cause sharp oscillations in the global star formation rates related to the $N$-body output times.

In can be seen in Fig. 10 that at low redshifts, the observational uncertainties quoted are mostly smaller than about 0.1 dex. If the Lilly et al. (1996) estimates are assumed to suffer a systematic error, then the apparently smooth behaviour of the other estimates would imply that the differences in the global star formation rates for the two merger/identity criteria are greater than the uncertainties in the low redshift observational estimates.

However, since the two rates approach each other as $z \rightarrow 0$ for any of the choice of metric parameters, the model difference again becomes smaller than the observational uncertainty.

Why should the global star formation rates for the two merger/identity criteria approach each other towards the present epoch? Given that the median merging percentages do not suddenly approach unity in the lowest redshift intervals (though they do start to increase slightly, see Table 2), this cannot be because matter is no longer thrown out into debris at late epochs. A possible explana- tion is the decrease in the contribution of low mass galaxies to the star formation rate at late times. In other words, not only is there less gas globally available for star formation at the late epochs, but a smaller fraction of this is contributed by the baryonic components of the smaller mass haloes than at earlier times.

So, an exact treatment of star formation in debris may be less important for zero redshift normalisation than for intermediate redshifts $(1 \lesssim z \lesssim 3)$.

\section{Discussion and conclusions}

One of the ways in which bottom-up hierarchical galaxy formation breaks the bottom-up hierarchy, i.e. the passage of matter through a "debris" phase, has been studied by varying the criterion for merging or identifying dark matter haloes between successive pure gravity $N$-body simulation output times, and by combining the resulting halo merging histories with semi-analytical formulae in order to see the effects on star formation.

An upper bound to the loss in star formation and in stars was modelled by using the median merging/identity criterion during construction of the halo merging history tree, i.e. by terminating haloes whose maximum merging percentages were below $2 P_{\text {med }}-1$, where $P_{\text {med }}$ is the median merging percentage for a given simulation and can be estimated by Eq. (5).

It was found that $P_{\text {med }}$ approaches unity as the redshift interval for comparing haloes increases or decreases away from $\Delta z \approx 0.4$, and that the median distances of debris are generally inside of the successor halo outermost radii. So, it is expected that the maximum possible dampening or truncation of star formation in matter that passes through the debris stage can only occur (in general) for a temporary period over a characteristic time scale (redshift interval) and that the matter contributes to the successor halo at later times. This is why the truncation of star formation and loss of stellar content modelled by use of the median merging/identity criterion provides an upper bound to possible losses.

The maximum fraction of matter passing through a debris stage, i.e. the minimum value of $P_{\text {med }}$, was found to be $P_{\text {med }} \approx 0.84$, i.e. about $16 \%$ of matter is in the debris stage for $\Delta z \sim 0.4$ and $z \sim 1$. (This fraction is only weakly dependent on the curvature parameters.)

The application of the median criterion was compared with the application of the largest successor merging/identity criterion, which provides a lower bound to star loss by linking every halo at a time step $t_{i}$ with its largest successor at $t_{i+1}$, unless no particles of the halo at $t_{i}$ are present in any halo at $t_{i+1}$.

The resulting losses for star formation and luminosity functions were found to be strongest for low luminosity galaxies and at intermediate redshifts $(1 \lesssim z \lesssim 3)$. The losses in both cases are mostly around 0.05 to 0.10 dex, i.e. $10 \%-30 \%$, have some dependence on the matter density parameter $\Omega_{0}$, and negligible dependence on the cosmological constant $\lambda_{0}$ (where $h \equiv 0.65$ is kept constant). 
This upper bound on likely losses in star formation rates and stellar populations is smaller than the uncertainties in estimates of corresponding observational parameters, so it may not be urgent to include a correction for this in Press-Schechter based galaxy formation models, provided that dwarf galaxy statistics are not of primary interest.

On the other hand, the modelling of higher order statistics such as the shape and slope of the faint end of the luminosity function or the evolution of galaxy morphology at intermediate redshifts could be expected to be more sensitive to the effects of debris. In modelling of these cases, care should be taken to cover parameter space which includes the redshift intervals for which maximum debris loss occurs ( $\Delta z \approx 0.4$ in the present study).

The two choices of merging/identity criterion adopted here bracket other cases in the literature. Since the values of $2 P_{\text {med }}-1$ were found to be well above $50 \%$, the $P=50 \%$ criterion adopted in Roukema et al. (1997) lies between the two cases studied.

The modified $P=50 \%$ criterion adopted by Kauffmann et al. (1999) is slightly stricter than the $P=$ $50 \%$ criterion of Roukema et al. (1997), due to the requirement regarding "merging" of the most bound particle of the progenitor halo. On the other hand, the later "recovery" of "lost" galaxies by those authors weakens the criterion, although not to the extent of the largest successor criterion, for which a single particle is sufficient to choose a successor halo (provided that no halo at $t_{i+1}$ contains two particles of the progenitor halo at $t_{i}$ ). So again this is intermediary between the cases studied here.

A useful possibility for following up the present work would be to study the possible positive effects of a debris stage on star formation rates, in particular to study the possibility that the creation of tidal dwarf galaxies could partly compensate for the negative effects of dampened or decreased star formation rates studied here. This would (ideally) require modelling of merging history trees that break the bottom-up hierarchy by including the possibility of multiple successor haloes (and galaxies). This would probably be more simple to implement in an $N$-body plus semi-analytical model rather than a Press-Schechter plus semi-analytical model.

In principle, it is possible that the creation of tidal dwarfs could partially compensate for the losses in star formation and stellar content constrained in the present study. However, since the fraction of debris which can even temporarily form highly dense tidal features, let alone permanently bound objects, seems to be no more than around $1 \%$ (Sect. 3.1.4) of a halo's mass, even at the epochs and for redshift intervals maximising debris loss, it is more likely that tidal dwarf haloes/galaxies only contribute to more subtle statistics representing galaxy formation.

Acknowledgements. Thanks to Ariane Lançon, Thanu Padmanabhan, Sunu Engineer and Jasjeet Bagla for helpful discussions, and the latter three for provision of pure gravity $N=2 \times 10^{6} N$-body simulations. This research has been

\section{ArFus (Arbre de fusion)}

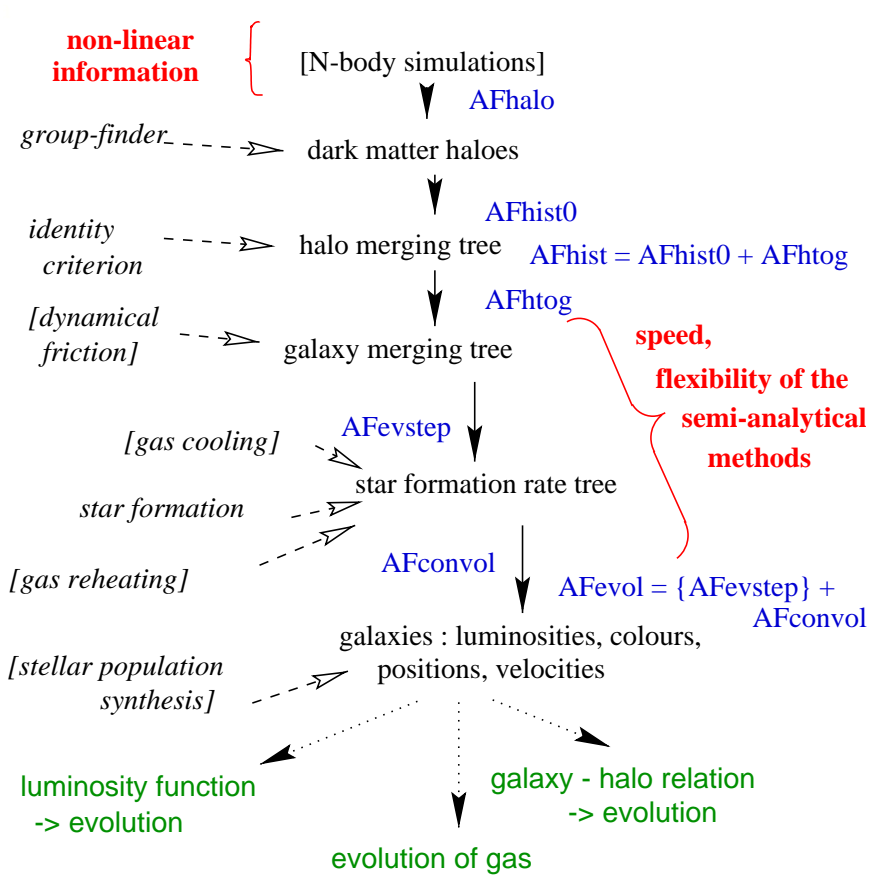

Fig. 12. Schematic flowchart of physical (in italics) and computational (in roman font) descriptions of the elements of the ARFus package. Key programme and subroutine names start with the prefix "AF". Square brackets indicate standard, nonoriginal contributions. Sans serif fonts (bottom) indicate examples of numerical output.

carried out with support from the Institut d'Astrophysique de Paris (CNRS), the Observatoire de Strasbourg, the EARA (European Association for Research in Astronomy) and the Polish Council for Scientific Research Grant KBN 2 P03D 01719.

\section{Appendix: The ArFus freeware package}

\subsection{General description}

The freeware (GNU Public License) package ARFus is an update of the galaxy formation modelling method of Roukema et al. (1993, 1997), and is intended to be a userfriendly software package. The initial releases are moderately user-friendly to anyone reasonably familiar with unix and fortran and moderately familiar with concepts in numerical galaxy formation modelling.

ARFUS is intended to be useful for generating merging history trees and evolving galaxy properties, and is available at http://www.iap.fr/users/roukema/ArFus/ index.html.

It combines the full non-linear information of the collapse of dark matter haloes from (pure gravity) $N$-body simulations with the flexibility and the rapidity of analytical formulae which represent gas thermodynamics and star formation in so-called "semi-analytical" galaxy formation models (Kauffmann et al. 1993; Cole et al. 1994). ARFUs is written primarily in Fortran 77 and should be 
easily compilable on any Unix machine. (Version ARFusV0.03 has been successfully compiled and run on DEC, SUN and linux platforms, and earlier versions also ran on IBM unix platforms.) The structure of the package is modularised into subroutines for the different logical and physical processes, and the major steps (detection of haloes, creation of halo merging tree, etc.) are run as individual programmes. The structure is shown schematically in Fig. 12. Documentation on the subroutines is automatically extracted directly from the compilable code and crosslinked in html format.

More details on the key subroutine AFhtog, which converts the halo merging history tree into a combined galaxy plus halo merging history tree and is called by AFhisto (which itself is called by a main programme AFhist), are provided in Sect. 5.2.

The choices of formulae for gas cooling and reheating (feedback) and star formation are focussed in special subroutines, so that these can be easily modified by the user. Parameter values are read in from text files, of which examples are provided in the package. The formulae and values used in the present study (by way of example) are mentioned in Sect. 2.4 above.

Different levels of use are possible depending on the user's interest:

(i) The simplest, black box, approach to the package would be to use output lists of galaxy absolute magnitudes and positions at desired output redshifts which have already been calculated from galaxy plus halo merging history trees derived from $N$-body simulations for standard cosmological parameter values and for "reasonable" options for other parameters;

(ii) A less trivial use of the package would be to enter in the pre-calculated halo plus galaxy merging trees, but to modify the input choices of star formation formulae, and then to generate lists of galaxy absolute magnitudes and positions at desired output redshifts;

(iii) An example of more sophisticated usage would be to derive halo merging history trees via ARFus from the user's own gravity-only $N$-body simulations.

\subsection{AFhtog: Conversion of a halo merging history tree into a galaxy plus halo merging history tree}

The most massive halo among a set of haloes which merge together between $t_{i}$ and $t_{i+1}$ to form a single halo contains a galaxy (unless no stars have formed), which is considered to be the "central" galaxy. Its halo is the "central" halo for this system. The other haloes initially contain galaxies, and are labelled "satellite" haloes. After the merger of a satellite halo with a central halo, the satellite galaxy will (in general) take some time before it merges with the central galaxy due to dynamical friction.

(0) (zeroth step) An initial galaxy+halo merging history tree (MHT) is created with one galaxy per halo at all times, i.e. galaxy merging is identical to halo merging.
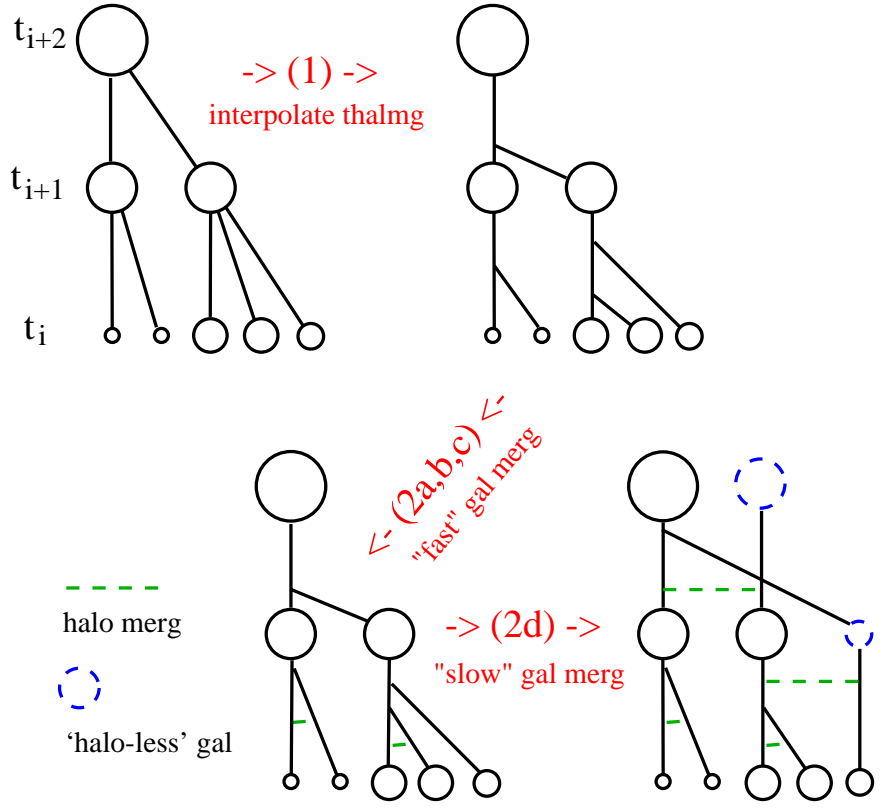

Fig. 13. Schematic description of the conversion of a halo merging history tree into a galaxy merging history tree. Circles represent haloes and/or galaxies, line segments represent identity and/or merging and time increases upwards. See 5.2 (1) to (2d) for details.

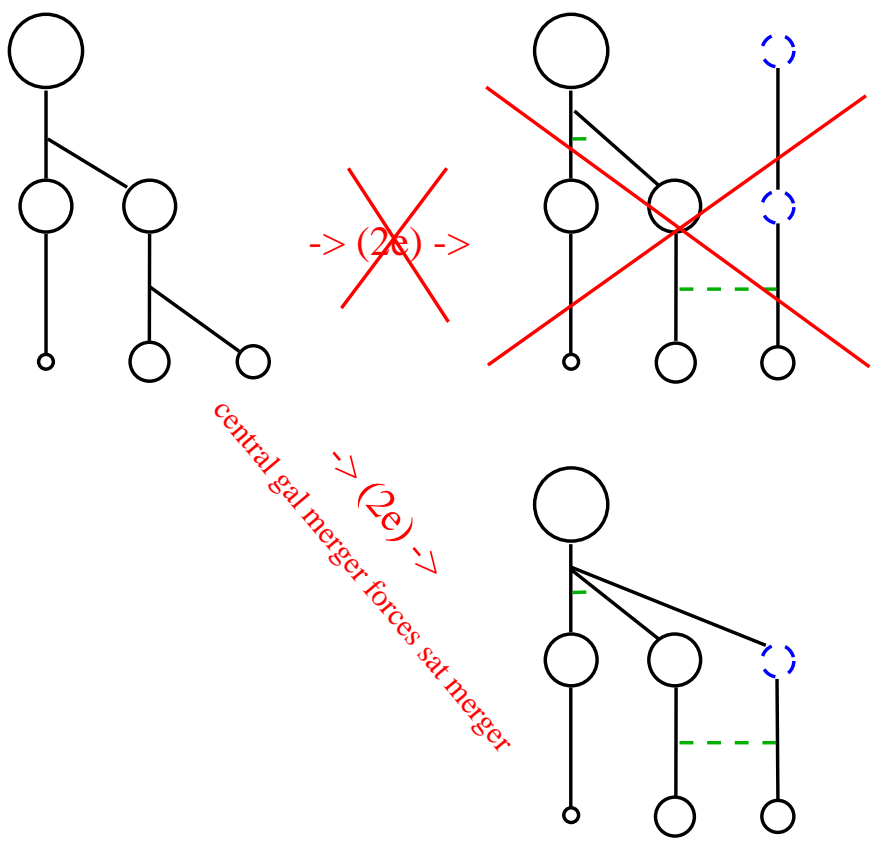

Fig. 14. Exceptional case requiring treatment by the algorithm which converts a halo merging history tree into a galaxy merging history tree. Symbols are as for Fig. 13. See 5.2 (2e) for details.

This is done by re-interpreting the variables representing the halo MHT to represent both a galaxy MHT and (initially) the halo MHT. When in later steps halo merging is distinguished from galaxy merging, a new variable (in effect, though not formally, a pointer) is added to enable the halo MHT to be represented via the galaxy MHT. 
The nodes of the halo MHT form a subset of the nodes of the galaxy MHT. In addition, other variables representing useful (but partly redundant) information on the combined galaxy+halo MHT are added.

The only subsequent modification to this is that when a satellite halo merges with a central halo, in general the galaxy which was in the satellite halo will not merge immediately with the central galaxy, so a "new" value of the galaxy/halo index i.e. a "new haloless" galaxy, is created to represent the satellite galaxy at each subsequent output time until the merger occurs. Physically, the satellite galaxy is orbiting within the central halo, but this link is represented indirectly. Since the galaxy/halo index of this galaxy only represents a galaxy, i.e. does not represent any halo during the period from $t_{i}$ to $t_{i+1}$, the galaxy is termed "halo-less". This is just an algorithmical convention, which distinguishes satellites which merge "fast" from those which merge "slowly" [see (c) and (d) below for definitions of "fast" and "slow"].

The following steps, illustrated schematically in Figs. 13 and 14 are then carried out.

(1) Halo merging times are interpolated between time steps, in order that halo merging does not just happen (in general) in multiple mergers at the ( $N$-body simulation) output time steps. (See Sect. 2.2.5 and Fig. 14 of Roukema et al. 1997 for more discussion.) An option is provided to have multiple mergers at the midpoints of the time interval instead of using the recommended isothermal potential interpolation.

(2) A loop through the output times from earliest to latest is carried out. At each output time, the following steps are carried out.

(a) A dynamical friction type formula is used to calculate how long, in principle, a satellite galaxy would take to merge with the central galaxy. This calculation is independent of the halo merging time calculated at step (1), for simplicity, except that the halo merging time is used to calculate the cosmological epoch. In the simplest case this time lapse is labelled $t_{\mathrm{g}}^{1}$.

(b) The calculated halo merger time $t_{\mathrm{h}}$ and $t_{\mathrm{g}}^{1}$ are used to estimate when the satellite galaxy should, in principle, merge with the central galaxy. (Note that both are time intervals, not cosmological times.)

(c) If a satellite would merge with the central galaxy before time $t_{i+1}$, then the linked parts of the MHT structure, which represent merging/identity between output times, do not need to be modified at all for that satellite galaxy and its halo. The fact that both the satellite halo and galaxy merge with the central halo and galaxy (in that order) during the interval between $t_{i}$ and $t_{i+1}$ is represented by $0<t_{\mathrm{h}}<t_{\mathrm{h}}+t_{\mathrm{g}}^{1}<t_{i+1}-t_{i}$.

This case is referred to as "fast" galaxy merging in Fig. 13.

(d) If a satellite would merge with the central galaxy after time $t_{i+1}$, then the linked parts of the MHT structure require modification, possibly several output times later. This is the first cause of the complexity of AFhist0.
This case is referred to as "slow" galaxy merging in Fig. 13. The difficulty is that a new "halo-less" galaxy needs to be added, which may take several time steps before finally merging with the central galaxy.

(e) The second cause of complexity is that in the same slow merging case (d) with a satellite galaxy $S_{1}$ and a central galaxy $C_{1}$, the central galaxy may itself become a satellite galaxy $C_{1}=S_{2}$ and merge with its own central galaxy $C_{2}$ at or before the time when the original satellite $\left(S_{1}\right)$ is calculated to merge. Because the dynamical friction type formula cannot be as good as (or identical with) the $N$-body simulation dynamics, it is possible that $S_{1}$ merges with $C_{1}=S_{2}$ after $C_{1}=S_{2}$ merges with $C_{2}$. Physically, while this is not totally impossible, it seems more reasonable, and is easier computationally, to decide that $S_{1}$ merges with $C_{2}$ at the time when $C_{1}=S_{2}$ merges with $C_{2}$. This is the option chosen here. That is, during the interval $\left(t_{i+j}, t_{i+j+1}\right)$ when $C_{1}=S_{2}$ merges with $C_{2}$, (i) the central galaxy of $S_{1}$ is redefined to be $C_{2}$ instead of $C_{1}=S_{2}$, and (ii) both $C_{1}=S_{2}$ and $S_{1}$ merge with $C_{2}$ at the same point of time during that time interval.

This case is shown schematically in Fig. 14. Although this case is rare, it needs to be treated algorithmically in one way or another if case (d) is allowed and if the galaxy MHT is to be logically complete.

If case (d) is not allowed, i.e. if all galaxy mergers are forced to occur before the output time $t_{i+1}$, then unless a very large $N$-body simulation output time step is chosen, the galaxy mergers would follow the halo mergers very quickly, so that little difference between the halo and galaxy MHT's would be possible.

If the galaxy MHT is not logically complete, then errors in the evaluation of the star formation history tree, such as reading or writing invalid bytes in memory would be possible.

Hence, inclusion of both cases (2d) and (2e) as outlined here seems necessary.

\section{References}

Benson, A. J., Cole, S., Frenk, C. S., Baugh, C. M., \& Lacey, C. G. 1999 [arXiv: astro-ph/9903343]

Blain, A. W., Smail, I., Ivison, R. J., \& Kneib, J.-P. 1999, MNRAS, 302, 632 [arXiv:astro-ph/9806062]

Bond, J. R., Cole, S., Efstathiou, G., \& Kaiser, N. 1991, ApJ, 379,440

Cole, S., Aragon-Salamanca, A., Frenk, C. S., Navarro, J. F., \& Zepf, S. E. 1994, MNRAS, 271, 781

Cole, S., \& Lacey, C. 1996, MNRAS, 281, 716

Connolly, A. J., Szalay, A. S., Dickinson, M., SubbaRao, M. U., \& Brunner, R. J. 1997, ApJ, 486, L11 [arXiv: astro-ph/9706255]

Davis, M., Efstathiou, G., Frenk, C. S., \& White, S. D. M. 1985, ApJ, 292, 371

Devriendt, J. E. G., Guiderdoni, B., \& Sadat, R. 1999, A\&A, 350, 381 [arXiv:astro-ph/9906332]

Dubinski, J., Mihos, J. C., \& Hernquist, L. 1996, ApJ, 462, 576 
Duc, P.-A., Brinks, E., Wink, J. E., \& Mirabel, I. F. 1997, A\&A, 326, 537

Duc, P.-A., \& Mirabel, I. F. 1994, A\&A, 289, 83

Efstathiou, G., Frenk, C. S., White, S. D. M., \& Davis, M. 1988, MNRAS, 235, 715

Farrar, K., \& Melott, A. L. 1990, Comp. Phys., 4, 185

Gallego, J., Zamorano, J., Aragón-Salamanca, A., \& Rego, M. 1996, ApJ, 459, L43

Governato, F., Baugh, C. M., Frenk, C. S., et al. 1998, Nature, 392,359

Gronwall, C. 1999, in Dwarf Galaxies and Cosmology, XVIII Moriond meeting, ed. T. X. Thuan, et al. (Gif-sur-Yvette: Éditions Frontières) [arXiv:astro-ph/9806240]

Guiderdoni, B., Hivon, E., Bouchet, F., \& Maffei, B. 1998, MNRAS, 295, 877

Hammer, F., \& Flores, H. 1999, in Dwarf Galaxies and Cosmology, XVIII Moriond meeting, ed. T. X. Thuan, et al. (Gif-sur-Yvette: Éditions Frontières) [arXiv: astro-ph/9806184]

Hernquist, L., \& Quinn, P. J. 1988, ApJ, 331, 682

Hernquist, L., \& Quinn, P. J. 1989, ApJ, 342, 1

Kauffmann, G., Colberg, J. M., Diaferio, A., \& White, S. D. M. 1999, MNRAS, 303, 188

Kauffmann, G., Nusser, A., \& Steinmetz, M. 1997, MNRAS, 286, 795

Kauffmann, G., White, S. D. M., \& Guiderdoni, B. 1993, MNRAS, 264, 201

Kennicutt, R. C. 1998, ApJ, 498, 541 [arXiv:astro-ph/ 9712213]

Klypin, A. A., Gottlöber, S., Kravtsov, A. V., \& Khokhlov, A. M. 1999, ApJ, 516, 530

Kravtsov, A. V., \& Klypin, A. A. 1999, ApJ, 520, 437

Lacey, C., \& Cole, S. 1993, MNRAS, 262, 627

Lanzoni, B., Mamon, G. A., \& Guiderdoni, B. 2000, MNRAS, 312,781

Lilly, S. J., Le Fèvre, O., Hammer, F., \& Crampton, D. 1996, ApJ, 460, L1

Loveday, J., Peterson, B. A., Efstathiou, G., \& Maddox, S. J. 1992, ApJ, 390, 338
Luminet, J.-P., \& Roukema, B. F. 1999, in Theoretical and Observational Cosmology, NATO Advanced Study Institute, Cargèse 1998, ed. M. Lachièze-Rey (Dordrecht, The Netherlands: Kluwer), 117 [arXiv:astro-ph/9901364]

Madau, P., Della Valle, M., \& Panagia, N. 1996, MNRAS, 283, 1388

Malin, D. F., Quinn, P. J., \& Graham, J. A. 1983, ApJ, 272, L5

Mendes de Oliveira, C., Plana, H., Amram, P., et al., in Dynamics of Galaxies: from the Early Universe to the Present, ed. F. Combes, G. A. Mamon, \& V. Charmandaris, San Francisco, USA: ASP, 353

Mihos, J. C., Dubinski, J., \& Hernquist, L. 1998, ApJ, 494, 183

Nagashima, M., \& Gouda, N. 1998, MNRAS, 301, 849 [arXiv: astro-ph/9608069]

Pettini, M., Kellogg, M., Steidel, C. C., et al. 1998, ApJ, 508, 539 [arXiv:astro-ph/9806219]

Press, W. H., \& Schechter, P. 1974, ApJ, 187, 425

Quinn, P. J. 1984, ApJ, 279, 596

Rodrigues, D. D. C., \& Thomas, P. 1996, MNRAS, 282, 361 [arXiv: astro-ph/9511018]

Roukema, B. F., Quinn, P. J., \& Peterson, B. A. 1993, in Observational Cosmology, ed. G. Chincarini et al., USA: ASP, 298 http://adsbit.harvard.edu/cgi-bin/ nph-iarticle_query?bibcode=1993obco . symp. . 298R

Roukema, B. F., Peterson, B. A., Quinn, P. J., \& RoccaVolmerange, B. 1997, MNRAS, 292, 835

Springel, V., \& White, S. D. M. 1999, MNRAS, 307, 162

Steidel, C. C., Adelberger, K. L., Giavalisco, M., Dickinson, M., \& Pettini, M. 1999, ApJ, 519, 1 [arXiv:astro-ph/9811399]

Sutherland, R. S., \& Dopita, M. A. 1993, ApJS, 88, 253

Tresse, L., \& Maddox, S. J. 1998, ApJ, 495, 691

Treyer, M.-A., Ellis, R. S., Milliard, B., Donas, J., \& Bridges, T. J. 1998, MNRAS, 300, 303

Toomre, A., \& Toomre, J. 1972, ApJ, 178, 623

Warren, M., Quinn, P. J., Salmon, J., \& Zurek, W. H. 1992, ApJ, 399, 405 